TITLE:

\title{
Generic Rigidity Matroids with Dilworth Truncations
}

\author{
$\operatorname{AUTHOR}(\mathrm{S})$ :
}

Tanigawa, Shin-ichi

\section{CITATION:}

Tanigawa, Shin-ichi. Generic Rigidity Matroids with Dilworth

Truncations. SIAM Journal on Discrete Mathematics 2012, 26(3): 14121439

ISSUE DATE:

2012-07-03

URL:

http://hdl.handle.net/2433/198582

RIGHT:

(C) 2012, Society for Industrial and Applied Mathematics 


\title{
GENERIC RIGIDITY MATROIDS WITH DILWORTH TRUNCATIONS*
}

\author{
SHIN-ICHI TANIGAWA ${ }^{\dagger}$
}

\begin{abstract}
We prove that the linear matroid that defines the generic rigidity of $d$-dimensional body-rod-bar frameworks (i.e., structures consisting of disjoint bodies and rods mutually linked by bars) can be obtained from the union of $\left(\begin{array}{c}d+1 \\ 2\end{array}\right)$ copies of a graphic matroid by applying variants of Dilworth truncation operations $n_{r}$ times, where $n_{r}$ denotes the number of rods. This result leads to an alternative proof of Tay's combinatorial characterizations of the generic rigidity of rod-bar frameworks and that of identified body-hinge frameworks.
\end{abstract}

Key words. rigidity matroids, rigidity of graphs, bar-joint frameworks, body-bar frameworks, count matroids

AMS subject classification. $52 \mathrm{C} 25$

DOI. $10.1137 / 100819473$

1. Introduction. One of the main topics in rigidity theory is to reveal a combinatorial characterization of the generic rigidity of frameworks. The celebrated Laman's theorem [18] asserts that a two-dimensional bar-joint framework (Figure 1.1(a)) is minimally rigid on a generic joint-configuration if and only if the graph $G=(V, E)$ obtained by regarding each joint as a vertex and each bar as an edge satisfies the following counting condition: $|E|=2|V|-3$ and $|F| \leq 2|V(F)|-3$ for any nonempty $F \subseteq E$, where $V(F)$ denotes the set of vertices spanned by $F$. However, in spite of exhausting efforts so far, the three-dimensional counterpart has not been obtained yet (see, e.g., [13, 41, 42]).

A common strategy to deal with a difficult problem in graph theory is to restrict a graph class, and several partial results are also known for the problem of characterizing three-dimensional generic rigidity for, e.g., triangulations [9, 41], bipartite graphs [38], sparse graphs [13], some minor closed classes [25], and the squares of graphs [16]. In rigidity theory, it is also reasonable to consider special types of structural models. Tay [30] considered a body-bar framework (Figure 1.1(b)) that consists of rigid bodies linked by bars. He proved that if we represent the underlying graph by identifying each vertex with each body and each edge with each bar, a body-bar framework is generically rigid in $\mathbb{R}^{3}$ if and only if the underlying graph contains six edge-disjoint spanning trees. Tay [31, 32] and Whiteley [39] independently proved that, even for the body-hinge models (Figure 1.1(c)), the same combinatorial characterization is true. Specifically, a body-hinge framework is a structure consisting of rigid bodies connected by hinges. Its underlying graph is represented by identifying each body with a vertex and each hinge with an edge. In this setting, the Tay-Whiteleys theorem asserts that a body-hinge framework is generically rigid in $\mathbb{R}^{3}$ if and only if the graph obtained by duplicating each edge by five parallel copies contains six edge-disjoint spanning

* Received by the editors December 28, 2010; accepted for publication (in revised form) July 9, 2012; published electronically September 27, 2012. The work was supported by Grant-in-Aid for JSPS Research Fellowships for Young Scientists.

http://www.siam.org/journals/sidma/26-3/81947.html

${ }^{\dagger}$ Research Institute for Mathematical Sciences, Kyoto University, 606-8502 Kyoto, Japan (tanigawa@kurims.kyoto-u.ac.jp). 


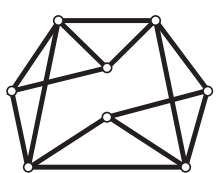

(a)

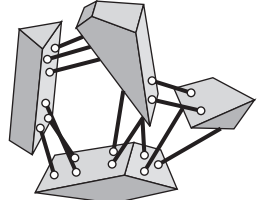

(b)

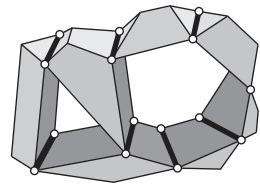

(c)

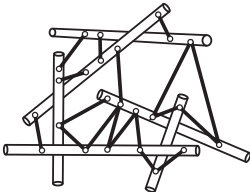

(d)

FIG. 1.1. (a) Two-dimensional bar-joint framework, (b) Body-bar framework, (c) Body-hinge framework, and (d) Rod-bar framework.

trees. Jackson and Jordán [14] further discuss the relation of generic rigidity of the body-bar-hinge model to the forest-packing problem in undirected graphs.

Although it is barely mentioned, Tay's work was actually done in a more general setting. An identified body-hinge framework is a body-hinge framework in which each hinge is allowed to connect more than two bodies. Historically, a combinatorial characterization of identified body-hinge frameworks was first conjectured by Tay and Whiteley in [34], and Tay affirmatively solved the conjecture in [31] as a by-product of his combinatorial characterization of rod-bar frameworks. A rod-bar framework is a structure consisting of disjoint rods linked by bars in $\mathbb{R}^{3}$ (Figure 1.1(d)). Each bar connects two rods, and each rod is allowed to be incident to several distinct bars. This structural model naturally comes up from body-bar frameworks by regarding each rod as a degenerated one-dimensional body.

Unfortunately, Tay's proof is based on a Henneberg-type graph construction with intricate and long analysis (the combinatorial part now follows from the recent result by Frank and Szegö [6]), and the combinatorics behind rigidity of rod-bar frameworks has not been understood well. To shed light on Tay's result, this paper provides a new proof of the combinatorial characterization of rod-bar frameworks.

We cope with a more general structural model, body-rod-bar frameworks, and prove that the linear matroid defining its generic rigidity is equal to a counting matroid defined on the underlying graphs (Theorem 4.9 and Corollary 4.14). Our proof technique is inspired by the idea of Lovász and Yemini given in [21]. They proved, as a new proof of Laman's theorem, that the linear matroid that defines the generic rigidity of two-dimensional bar-joint frameworks can be obtained from the union of two copies of a graphic matroid by Dilworth truncation. Roughly speaking, Dilworth truncation is an operation to construct a new linear matroid from an old one by restricting the domain of entries of each vector to a generic hyperplane. (See subsection 2.4 for the definition.) The main difference between our situation and that of Lovász and Yemini is that we need to apply such truncation operations more than once (while they used it only once). Indeed, it is not trivial to keep up the representation of the resulting matroid when applying Dilworth truncation operations several times, as each hyperplane must be inserted in a "generic" position relative to the preceding hyperplanes. We will overcome the difficulty by extending an idea of Lovász [20] so that each truncation is performed within a designated subspace.

A bar-joint framework can be considered as a body-bar framework consisting of zero-dimensional bodies. As combinatorial properties of body-bar frameworks with three-dimensional bodies are well understood $[30,37,39]$ in $\mathbb{R}^{3}$, it is then natural to consider body-bar frameworks with one-dimensional bodies (i.e., rods) toward a combinatorial characterization of bar-joint frameworks. Our proof explicitly describes how each three-dimensional body can be replaced by a one-dimensional body by the use of truncations. 
The paper is organized as follows. In section 2, we first review (poly)matroids induced by submodular functions and then review two classical techniques proposed by Lovász [20]: the first one shows how to obtain a maximum matroid from a polymatroid defined by a family of flats in projective space, and the second one is Dilworth truncation. In section 3, we provide a proof of a combinatorial characterization of body-bar frameworks by Tay [30] from the viewpoint of matroids of flat families (discussed in section 2). Our main result is section 4, where we prove a combinatorial characterization of body-rod-bar frameworks. In section 5, we will discuss identified body-hinge frameworks and several unsolved problems. As another application of the Dilworth truncation, in section 6, we provide a direct proof of the combinatorial characterization of $d$-dimensional direction-rigidity given by Whiteley [41, Theorem 8.2.2]. We believe that our proof technique is so powerful that it can be applied to a wider range of truncated matroids appeared in combinatorial geometry (see, e.g., [41]).

We conclude this introduction by listing some notation used throughout the paper. For a vector space $W=\mathbb{R}^{k}$, let $\mathbb{P}(W)$ denote the projective space $\mathbb{P}^{k-1}$ associated with $W$. For a vector $\boldsymbol{v}=\left(v^{1}, \ldots, v^{k}\right) \in W$, the projective point associated with $\boldsymbol{v}$ is denoted by $[\boldsymbol{v}]=\left[v^{1}, \ldots, v^{k}\right] \in \mathbb{P}(W)$. For a flat $A$ in $\mathbb{P}(W)$, the rank of $A$ is defined by $\operatorname{rank}(A)=\operatorname{dim} W^{\prime}$, where $W^{\prime}$ is the linear subspace of $W$ associated with $A$. For a finite family $\mathcal{A}$ of flats, the span of $\mathcal{A}$ is denoted by $\overline{\mathcal{A}}$. $\mathcal{A}$ is called disconnected if there is a partition $\left\{\mathcal{A}_{1}, \mathcal{A}_{2}\right\}$ of $\mathcal{A}$ into nonempty subsets such that $\operatorname{rank}(\overline{\mathcal{A}})=\sum_{i=1,2} \operatorname{rank}\left(\overline{\mathcal{A}_{i}}\right)$ (equivalently, $\overline{\mathcal{A}_{1}} \cap \overline{\mathcal{A}_{2}}=\emptyset$ ). Otherwise $\mathcal{A}$ is said to be connected. (Note that a singleton set is connected.)

We consider a finite graph $G=(V, E)$ that may contain parallel edges but no loop. If $G$ has neither parallel edges nor a loop, $G$ is said to be simple. We sometimes use $V(G)$ and $E(G)$ to denote the sets of vertices and edges of $G$, respectively. For $v \in V$, let $\delta_{G}(v)$ be the set of edges incident to $v$ in $G$. We say that $F \subseteq E$ spans $v \in V$ if $v$ is incident to some edge of $F$. For $F \subseteq E, V(F)$ denotes the set of vertices spanned by $F$.

\section{Preliminaries.}

2.1. Polymatroids. Let $E$ be a finite set. A function $\mu: 2^{E} \rightarrow \mathbb{R}$ is called submodular if $\mu(X)+\mu(Y) \geq \mu(X \cup Y)+\mu(X \cap Y)$ for every $X, Y \subseteq E$. $\mu$ is called monotone if $\mu(X) \leq \mu(Y)$ for every $X \subseteq Y$.

Suppose $\mu: 2^{E} \rightarrow \mathbb{Z}$ is an integer-valued function on $E$ satisfying $\mu(\emptyset)=0$. The pair $(E, \mu)$ is called a polymatroid if $\mu$ is monotone and submodular, and $\mu$ is called the rank function of $(E, \mu)$. It is particularly called a matroid if $\mu$ further satisfies $\mu(e) \leq 1$ for every $e \in E . F \subseteq E$ is called independent if $|F|=\mu(F)$, and a maximal independent set and a minimal dependent set are called a base and a circuit, respectively. An element $e \in E$ is called a coloop if every base contains $e$.

2.2. Submodular functions and induced polymatroids. Suppose $\mu: 2^{E} \rightarrow$ $\mathbb{Z}$ is a monotone submodular function such that $\mu(F) \geq 0$ for every nonempty $F \subseteq E$ (but $f(\emptyset)<0$ is allowed). We define $\hat{\mu}: 2^{E} \rightarrow \mathbb{Z}$ by

$$
\hat{\mu}(F)=\min \left\{\sum_{i=1}^{k} \mu\left(F_{i}\right)\right\} \quad(F \subseteq E),
$$

where the minimum is taken over all partitions $\left\{F_{1}, \ldots, F_{k}\right\}$ of $F$ into nonempty subsets. It is known that $\hat{\mu}$ is a monotone submodular function satisfying $\hat{\mu}(\emptyset)=0$ (see, e.g., $[28$, Chapter 48$]$ or [7]), and hence the pair $(E, \hat{\mu})$ forms a polymatroid. It is also known that $\hat{\mu}$ is the unique largest among all monotone submodular functions $\mu^{\prime}$ satisfying $0 \leq \mu^{\prime}(F) \leq \mu(F)$ for each $F \subseteq E$. 
Edmonds and Rota [3] observed that a monotone submodular function $\mu: 2^{E} \rightarrow \mathbb{Z}$ induces a matroid $\left(E, r_{\mu}\right)$ on $E$, where $F \subseteq E$ is independent if and only if $\left|F^{\prime}\right| \leq \mu\left(F^{\prime}\right)$ for every nonempty $F^{\prime} \subseteq F$ (see also [27]). Observe that this matroid takes the maximum rank among those satisfying $r_{\mu}(F) \leq \min \{\mu(F),|F|\}$ for every nonempty $F \subseteq E$, and indeed the rank function $r_{\mu}$ can be written as

$$
r_{\mu}(F)=\min _{F_{0} \subseteq F}\left\{\left|F_{0}\right|+\hat{\mu}\left(F \backslash F_{0}\right)\right\} \quad(F \subseteq E),
$$

(see, e.g., [28, section 44.6a]). Namely,

$$
r_{\mu}(F)=\min \left\{\left|F_{0}\right|+\sum_{i=1}^{k} \mu\left(F_{i}\right)\right\} \quad(F \subseteq E),
$$

where the minimum is taken over all partitions $\left\{F_{0}, F_{1}, \ldots, F_{k}\right\}$ of $F$ such that $F_{1}, \ldots, F_{k}$ are nonempty (and $F_{0}=\emptyset$ is allowed). Geometric interpretations of these results will be discussed in the next two subsections. More detailed descriptions on general (poly)matroids can be found in, e.g., [28, 7, 26].

2.3. Generic matroids. Let $E$ be a finite set. We associate each element $e \in E$ with a flat $A_{e}$ in a real projective space and let $\mathcal{A}=\left\{A_{e}: e \in E\right\}$. Also, for $F \subseteq E$, we denote $\left\{A_{e} \in \mathcal{A}: e \in F\right\}$ by $\mathcal{A}_{F}$. If we define a rank function $\operatorname{rank}_{\mathcal{A}}: 2^{E} \rightarrow \mathbb{Z}$ by $\operatorname{rank}_{\mathcal{A}}(F)=\operatorname{rank}\left(\overline{\mathcal{A}_{F}}\right)$ for $F \subseteq E$, the pair $\left(E, \operatorname{rank}_{\mathcal{A}}\right)$ forms a linear polymatroid, which is denoted by $\mathcal{P} \mathcal{M}(\mathcal{A})$. A polymatroid turns out to be a matroid by bounding the rank of each element by one. Below, we review a geometric method for getting a maximum linear matroid from the linear polymatroid $\mathcal{P} \mathcal{M}(\mathcal{A})$.

We shall associate a representative point $x_{e} \in A_{e}$ with each $A_{e} \in \mathcal{A}$. Let us denote $\left\{x_{e}: e \in E\right\}$ by $X$. The set $X$ of representative points is said to be in generic position if, for every $X^{\prime} \subseteq X$ and for every $x_{e} \in X^{\prime}$,

$$
x_{e} \in \overline{X^{\prime}-x_{e}} \Rightarrow A_{e} \subseteq \overline{X^{\prime}-x_{e}} .
$$

It is not difficult to see that, for any finite flat family $\mathcal{A}$, the set $X$ of representative points can be taken to be in generic position. For any $x_{e} \in X, A_{e} \backslash \bigcup\left\{\overline{X^{\prime}}: X^{\prime} \subseteq\right.$ $X-x_{e}$ with $\left.A_{e} \not \subset \overline{X^{\prime}}\right\}$ forms a dense open subset of $A_{e}$; hence, if $x_{e} \in \overline{X^{\prime}}$ for some $X^{\prime} \subseteq X-x_{e}$ with $A_{e} \not \subset \overline{X^{\prime}}$, then by continuously (and slightly) moving $x_{e}$ on $A_{e}$ it can avoid $\overline{X^{\prime}}$ without creating a new violation for generic position.

For $F \subseteq E$, the dimension of the linear subspace spanned by $\left\{x_{e}: e \in F\right\}$ is defined as the rank of $F$ (with respect to $X$ ), and we denote it by $\operatorname{rank}_{X}(F)$, i.e., $\operatorname{rank}_{X}(F)=\operatorname{rank}\left(\overline{\left\{x_{e}: e \in F\right\}}\right)$. The linear matroid $\left(E, \operatorname{rank}_{X}\right)$ is called a matroid associated with $\mathcal{A}$.

Theorem 2.1 (Lovász [20]). Let $\mathcal{A}=\left\{A_{e}: e \in E\right\}$ be a finite family of flats, and let $X$ be a set of representative points of $\mathcal{A}$ in generic position. Then,

$$
\operatorname{rank}_{X}(E)=\min _{F \subseteq E}\left\{|E \backslash F|+\operatorname{rank}\left(\overline{\mathcal{A}_{F}}\right)\right\} .
$$

By restricting the argument to $F \subseteq E$, we also have $\operatorname{rank}_{X}(F)=\min _{F^{\prime} \subseteq F}\{\mid F \backslash$ $\left.F^{\prime} \mid+\operatorname{rank}\left(\overline{\mathcal{A}_{F^{\prime}}}\right)\right\}$. The rank of the linear matroid associated with $\mathcal{A}$ does depend on the choice of $X$. However, Theorem 2.1 implies that it attains the maximum and is invariant when $X$ is in generic position. (Notice that the $\leq$ direction of (2.5) holds even though $X$ is not in generic position. For any $F \subseteq E, \operatorname{rank}_{X}(E) \leq \operatorname{rank}_{X}(E \backslash$ $F)+\operatorname{rank}_{X}(F) \leq|E \backslash F|+\operatorname{rank}\left(\left\{A_{e} \in \mathcal{A}: e \in F\right\}\right)$.) This motivates us to define the generic matroid. The generic matroid associated with $\mathcal{A}$, denoted $\mathcal{M}(\mathcal{A})$, is defined to be $\mathcal{M}(\mathcal{A})=\left(E, \operatorname{rank}_{X}\right)$ with $X$ in generic position. 
2.4. Dilworth truncation. Let $\mathcal{A}$ be a finite set of flats. We now consider restricting flats of $\mathcal{A}$ to a generic hyperplane. A hyperplane $H$ is called generic relative to $\mathcal{A}$ if it satisfies the following condition: ${ }^{1}$; for any $A_{1}, A_{2} \in \mathcal{A}$ and any $\mathcal{F} \subseteq\{A \cap H: A \in \mathcal{A}\}$,

$$
\overline{\left(A_{1} \cap H\right) \cup \mathcal{F}} \cap \overline{\left(A_{2} \cap H\right) \cup \mathcal{F}} \neq \overline{\mathcal{F}} \quad \Rightarrow \quad \overline{A_{1} \cup \mathcal{F}} \cap \overline{A_{2} \cup \mathcal{F}} \not \subset H .
$$

Although the details are omitted, it can be verified that almost all hyperplanes are generic relative to $\mathcal{A}$. For a family $\mathcal{A}$ of flats and a hyperplane $H$, we shall abbreviate $\{A \cap H: A \in \mathcal{A}\}$ as $\mathcal{A} \cap H$. The following result was also done by Lovász [20].

THeOREM 2.2 (Lovász [20]). Let $\mathcal{A}$ be a finite family of flats in a real projective space and $H$ be a generic hyperplane relative to $\mathcal{A}$. Then,

$$
\operatorname{rank}(\overline{\mathcal{A} \cap H})=\min \left\{\sum_{i=1}^{k}\left(\operatorname{rank}\left(\overline{\mathcal{A}_{i}}\right)-1\right)\right\},
$$

where the minimum is taken over all partitions $\left\{\mathcal{A}_{1}, \ldots, \mathcal{A}_{k}\right\}$ of $\mathcal{A}$ into nonempty subsets.

This operation (of restricting flats to a generic hyperplane) is referred to as Dilworth truncation. Indeed, as noted in [28], Theorems 2.1 and 2.2 provide geometric interpretations of the formulae (2.1) and (2.3) for linear polymatroids.

The same result was also obtained by Mason [23, 22] from the view point of combinatorial geometry (projective matroids). The papers of Mason [23, 22] include examples of Dilworth truncation.

2.5. $\boldsymbol{M}$-connectivity and $\boldsymbol{P}$-connectivity. Let $\mathcal{M}=(E, r)$ be a matroid on a finite set $E$ with the rank function $r$. A subset $F \subseteq E$ is called $M$-connected if for any pair $e, e^{\prime} \in F, F$ has a circuit of $\mathcal{M}$ that contains $e$ and $e^{\prime}$. For simplicity of the description, a singleton $\{e\}$ is also considered as an $M$-connected set. A maximal $M$-connected set is called an $M$-connected component. It is well know that the union of two $M$-connected sets is $M$-connected if their intersection is nonempty, and thus $E$ is uniquely partitioned into $M$-connected components $E_{1}, \ldots, E_{k}$ (see, e.g., [26, Chapter 4]). Since there is no circuit intersecting two components, we have $r(E)=$ $\sum_{i=1}^{k} r\left(E_{i}\right)$. Alternatively, we can use it for the definition of $M$-connectivity: $F \subseteq E$ is $M$-connected if and only if there is no partition $\left\{F_{1}, \ldots, F_{k}\right\}$ of $F$ into at least two nonempty subsets such that $r(F)=\sum_{i=1}^{k} r\left(F_{i}\right)$.

The concept of connectivity can be extended to polymatroids. Let $\mathcal{P} \mathcal{M}=(E, \mu)$ be a polymatroid on a finite set $E$. Then, $F \subseteq E$ is said to be $P$-connected if there is no partition $\left\{F_{1}, \ldots, F_{k}\right\}$ of $F$ into at least two nonempty subsets such that $\mu(F)=\sum_{i=1}^{k} \mu\left(F_{i}\right)$. A maximal $P$-connected set is called a $P$-connected component. The union of two $P$-connected sets is $P$-connected if their intersection is nonempty, and thus $E$ is uniquely partitioned into $P$-connected components. If we consider linear polymatroids, the concept of $P$-connectivity coincides with the connectivity of flats we introduced in the introduction.

\footnotetext{
${ }^{1}$ Lovász claimed Theorem 2.2 with a much weaker assumption. He defined that a hyperplane $H$ is generic if for any subsets $X, Y$, and $Z$ of $\mathcal{A}$ satisfying $\overline{(X \cap H) \cup Y} \cap \overline{(X \cap H) \cup Z} \subseteq H$, we have $\overline{(X \cap H) \cup Y} \cap \overline{(X \cap H) \cup Z} \subseteq \overline{X \cap H}$. Theorem 2.2, however, fails in this setting. For example, suppose the underlying projective space is three-dimensional, and $\mathcal{A}$ consists of three distinct hyperplanes $\left\{A_{1}, A_{2}, A_{3}\right\}$ such that $A_{1} \cap A_{2}=A_{2} \cap A_{3}=A_{3} \cap A_{1}$ is a line $l$. If we take $H$ as a hyperplane distinct from $A_{i}$ but containing $l, H$ satisfies the condition to be generic. However, the left-hand side of (2.7) is $\operatorname{rank}(\overline{\{A \cap H: A \in \mathcal{A}\}})=\operatorname{rank}(l)=2$, while the right-hand side is equal to $\operatorname{rank}\left(\overline{\left\{A_{1}, A_{2}, A_{3}\right\}}\right)-1=3$.
} 
A $P$-connected set (and similarly, an $M$-connected set) is called trivial if it is singleton; otherwise it is nontrivial.

3. Body-bar frameworks. A body-bar framework is a structure consisting of rigid bodies linked by bars (Figure 1.1(b)). The generic rigidity of body-bar frameworks is characterized by Tay [30] (and a simpler proof was given by Whiteley [39]). In this section, we present a proof of this characterization from the viewpoint of matroids of flat families. In the subsequent sections, $d$ denotes the dimension of frameworks, and let $D=\left(\begin{array}{c}d+1 \\ 2\end{array}\right)$.

3.1. Union of copies of graphic matroid. We first review the union of copies of graphic matroid to which Tay related the generic rigidity matroid in the body-bar model.

3.1.1. Graphic matroid. Let $G=(V, E)$ be a finite undirected graph. We denote the graphic matroid of $G$ by $\mathcal{G}(G)$, that is, the matroid induced by the monotone submodular function $g: 2^{E} \rightarrow \mathbb{Z}$ defined by $g(F)=|V(F)|-1$ for $F \subseteq E$. Namely, $F \subseteq E$ is independent in $\mathcal{G}(G)$ if and only if $|F| \leq|V(F)|-1$ for nonempty $F \subseteq E$, and equivalently $F$ is a forest.

Let $I(G)=\left[a_{i j}\right]$ be the incidence matrix of a digraph obtained from $G$ by arbitrary assigning a direction to each edge, i.e,

$$
a_{i j}= \begin{cases}1 & \text { if vertex } v_{j} \text { is the tail of } \operatorname{arc} e_{i}, \\ -1 & \text { if vertex } v_{j} \text { is the head of } \operatorname{arc} e_{i}, \\ 0 & \text { otherwise }\end{cases}
$$

It is well known that $\mathcal{G}(G)$ is linear as it is represented by the row matroid of $I(G)$.

3.1.2. Graphic matroid union. For a matroid $\mathcal{M}=(E, \mathcal{I})$ with a collection $\mathcal{I}$ of independent sets, the union of $D$ independent sets, i.e., $\left\{I_{1} \cup \cdots \cup I_{D}: I_{i} \in\right.$ $\mathcal{I}, i=1, \ldots, D\}$, again forms the collection of independent sets of a matroid. This matroid is called the union of $D$ copies of $\mathcal{M}$. In the union of $D$ copies of the graphic matroid, denoted $D \mathcal{G}(G), F \subseteq E$ is independent if and only if $F$ can be partitioned into $D$ edge-disjoint forests. $D \mathcal{G}(G)$ is indeed the matroid induced by the monotone submodular function $D g:=D(|V(\cdot)|-1)$ defined on $E$ [24]. This implies that $E$ can be partitioned into $D$ edge-disjoint spanning trees if and only if $|E|=D(|V|-1)$ and $|F| \leq D(|V(F)|-1)$ for any nonempty $F \subseteq E$.

It is also known that $D \mathcal{G}(G)$ can be represented as a row linear matroid by introducing indeterminates. For each integer $k$ with $1 \leq k \leq D$, let $I^{k}=\left[a_{i j}^{k}\right]$ be a $|E| \times|V|$-matrix defined by

$$
a_{i j}^{k}= \begin{cases}\alpha_{e_{i}}^{k} & \text { if vertex } v_{j} \text { is the tail of } \operatorname{arc} e_{i} \\ -\alpha_{e_{i}}^{k} & \text { if vertex } v_{j} \text { is the head of } \operatorname{arc} e_{i} \\ 0 & \text { otherwise }\end{cases}
$$

where $\alpha_{e}^{k}$ 's are algebraically independent indeterminates over $\mathbb{Q}$. Denote the $|E| \times$ $D|V|$-matrix $\left[I^{1}\left|I^{2}\right| \ldots \mid I^{D}\right]$ by $D I(G)$. Then, $D \mathcal{G}(G)$ is represented by $D I(G)$ (see, e.g., $[23,39])$.

This representation gives us another way to look at $D \mathcal{G}(G)$. We associate a $D$ dimensional vector space $V_{u}=\mathbb{R}^{D}$ with each vertex $u$ in the subsequent discussion, and $V_{V}$ denotes the direct product of $V_{u}$ for all $u \in V$. In $D I(G)$, the row associated with an edge $e=u v$ is represented by 


$$
(0, \cdots, 0, \overbrace{\alpha_{e}^{1}, \ldots, \alpha_{e}^{D}}^{u}, 0, \cdots, 0, \overbrace{-\alpha_{e}^{1}, \ldots,-\alpha_{e}^{D}}^{v}, 0, \cdots, 0),
$$

where we changed the column ordering so that the entries associated with each vertex form a block (and throughout the subsequent discussions we will refer to this ordering). When looking at $\alpha_{e}^{1}, \ldots, \alpha_{e}^{D}$ as independent parameters in $\mathbb{R}$, the space spanned by vectors (3.1) form a $D$-dimensional vector space contained in $V_{u} \times V_{v}$. We can identify this $D$-dimensional vector space with a $(D-1)$-dimensional flat in $\mathbb{P}\left(V_{V}\right)$. We denote this flat by $A_{e}$ and let $\mathcal{A}:=\left\{A_{e}: e \in E\right\}$. Then, $D \mathcal{G}(G)$ can be considered as the generic matroid $\mathcal{M}(\mathcal{A})$ associated with $\mathcal{A}$.

\subsection{Generic body-bar matroids.}

3.2.1. Plücker coordinates. Throughout the paper, let $W=\mathbb{R}^{d+1}$. For simplicity, we shall use the standard basis $e_{1}, \ldots, e_{d+1}$ of $W=\mathbb{R}^{d+1}$ and use the dot product as an inner product. Also $W$ is identified with its dual.

Recall that the exterior product $\bigwedge^{k} W$ of degree $k$ is a $\left(\begin{array}{c}d+1 \\ k\end{array}\right)$-dimensional vector space and can be naturally identified with $\mathbb{R}^{\left(\begin{array}{c}++1 \\ 2\end{array}\right)}$ by associating $e_{i_{1}} \wedge \cdots \wedge e_{i_{k}}$ with an element of the standard basis of $\mathbb{R}^{\left(\begin{array}{c}d+1 \\ k\end{array}\right)}$ for each $1 \leq i_{1}<\cdots<i_{k}<d+1$. In particular, $\bigwedge^{2} W=\mathbb{R}^{D}$.

The collection of $k$-dimensional subspaces in $W$ is called the Grassmannian, denoted $\operatorname{Gr}(k, W)$. The Plücker embedding $p: G r(k, W) \rightarrow \mathbb{P}\left(\bigwedge^{k} W\right)$ is a bijection between $k$-dimensional vector spaces $X \in G r(k, W)$ and projective equivalence classes $\left[v_{1} \wedge \cdots \wedge v_{k}\right] \in \mathbb{P}\left(\wedge^{k} W\right)$ of decomposable elements, where $\left\{v_{1}, \ldots, v_{k}\right\}$ is a basis of $X$. In the subsequent discussions, we shall identify $\operatorname{Gr}(k, W)$ and its image of the Plücker embedding and regard $G r(k, W)$ as a subset of $\mathbb{P}\left(\bigwedge^{k} W\right)$.

It is well known that each point of $G r(k, W)$ can be coordinatized by the so-called Plücker coordinate once we fix a basis of $W$. If a basis $\left\{v_{1}, \ldots, v_{k}\right\}$ of $X \in G r(k, W)$ is represented by $v_{i}=\sum_{j=1}^{d+1} p_{i j} e_{j}$ with the $k \times(d+1)$-matrix $P=\left[p_{i j}\right]$, then we have

$$
v_{1} \wedge \cdots \wedge v_{k}=\sum_{i_{1}<\cdots<i_{k}} \operatorname{det} P_{i_{1}, \ldots, i_{k}} e_{i_{1}} \wedge \cdots \wedge e_{i_{k}},
$$

where $P_{i_{1}, \ldots, i_{k}}$ is the $k \times k$-submatrix of $P$ consisting of $i_{j}$ th columns. Let us simply denote $p_{i_{1}, \ldots, i_{k}}=\operatorname{det} P_{i_{1}, \ldots, i_{k}}$. The ratio of $p_{i_{1}, \ldots, i_{k}}$ for $1 \leq i_{1}<\cdots<i_{k} \leq d+1$ is called the Plücker coordinate of $X$.

It is well known that $\left[p_{i, j}\right]_{1 \leq i<j \leq d+1} \in \mathbb{P}\left(\bigwedge^{2} W\right)$ is in $\operatorname{Gr}(2, W)$ if and only if $p_{i, j} p_{k, l}-p_{i, k} p_{j, l}+p_{i, l} p_{j, k}=0$ for $1 \leq i<j<k<l \leq d+1$, and $\operatorname{Gr}(2, W)$ is an irreducible quadratic variety (see, e.g., [10]). In particular, if $d=3, \operatorname{Gr}(2, W)$ is a nonsingular quadratic variety written by

$$
\left\{\left[p_{i, j}\right]_{1 \leq i<j \leq 4} \in \mathbb{P}\left(\bigwedge^{2} W\right): p_{1,2} p_{3,4}-p_{1,3} p_{2,4}+p_{1,4} p_{2,3}=0\right\} .
$$

Through the one-to-one correspondence between a $k$-dimensional linear subspace and its orthogonal complement, $\operatorname{Gr}(d-1, W)$ is also an irreducible quadratic variety in $\mathbb{P}\left(\bigwedge^{d-1} W\right)$ described in the same form as $\operatorname{Gr}(2, W)$.

Let us define a product $\langle\cdot, \cdot\rangle: \bigwedge^{k} W \times \bigwedge^{d+1-k} W \rightarrow \mathbb{R}$ by

$$
\langle\boldsymbol{p}, \boldsymbol{q}\rangle=\sum_{i_{1}<\cdots<i_{k}}(-1)^{i_{1}+\cdots+i_{k}} p_{i_{1}, \ldots, i_{k}} q_{j_{1}, \ldots, j_{d+1-k}}
$$

for $\boldsymbol{p}=\left(p_{i_{1} \ldots i_{k}}\right) \in \bigwedge^{k} W$ and $\boldsymbol{q}=\left(q_{i_{1} \ldots i_{d+1-k}}\right) \in \bigwedge^{d+1-k} W$, where $j_{1}, \ldots, j_{d+1-k}$ are the complement of $i_{1}, \ldots, i_{k}$ in $[d+1]$ with $j_{1}<\cdots<j_{d+1-k}$. For example, for $d=3$ 
and $k=2$, we have $\langle\boldsymbol{p}, \boldsymbol{q}\rangle=p_{1,2} q_{3,4}-p_{1,3} q_{2,4}+p_{1,4} q_{2,3}+p_{2,3} q_{1,4}-p_{2,4} q_{1,3}+p_{3,4} q_{1,2}$. In general, it has the following useful property: a $k$-dimensional linear subspace $X$ and a $(d+1-k)$-dimensional linear subspace $Y$ have a nonzero intersection if and only if the corresponding Plücker coordinates $[\boldsymbol{p}]$ and $[\boldsymbol{q}]$ satisfy $\langle\boldsymbol{p}, \boldsymbol{q}\rangle=0$. This is because if $\boldsymbol{p}$ and $\boldsymbol{q}$ are decomposable, then $\langle\boldsymbol{p}, \boldsymbol{q}\rangle$ is the determinant of a square matrix obtained by aligning composition elements of $\boldsymbol{p}$ and $\boldsymbol{q}$.

This product can be seen as a dot product in $\mathbb{R}^{\left(\begin{array}{c}d+1 \\ k\end{array}\right)}$ through the so-called Hodge star-operator. The Hodge star-operator is a linear operation $*: \bigwedge^{k} W \rightarrow \bigwedge^{d+1-k} W$ defined by

$$
*\left(e_{i_{1}} \wedge \cdots \wedge e_{i_{k}}\right)=\operatorname{sign}(\sigma) e_{j_{1}} \wedge \cdots \wedge e_{j_{d+1-k}},
$$

where $j_{1}, \ldots, j_{d+1-k}$ are the complement of $i_{1}, \ldots, i_{k}$ in $[d+1]$ and $\operatorname{sign}(\sigma)$ denotes the sign of the permutation $\sigma=\left(\begin{array}{cccccc}i_{1} & \ldots & i_{k} & j_{1} & \ldots & j_{d+1-k} \\ 1 & \ldots & k & k+1 & \ldots & d+1\end{array}\right)$. For example, if $d=3$ and $k=2, * \boldsymbol{q}=\left(q_{3,4},-q_{2,4}, q_{2,3}, q_{1,4},-q_{1,3}, q_{1,2}\right)$ for $\boldsymbol{q}=\left(q_{1,2}, q_{1,3}, q_{1,4}, q_{2,3}\right.$, $\left.q_{2,4}, q_{3,4}\right)$.

By identifying $\bigwedge^{k} W$ with $\bigwedge^{d+1-k} W$ through $*$ and identifying $\bigwedge^{k} W$ with $\mathbb{R}^{\left(\begin{array}{c}d+1 \\ k\end{array}\right)}$

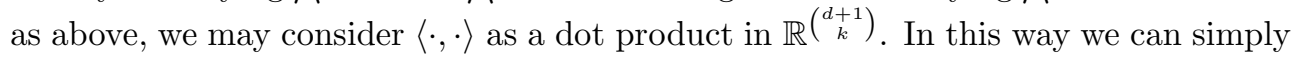
consider a product between $\bigwedge^{k} W$ and $\bigwedge^{d+1-k} W$, where $\boldsymbol{p} \cdot \boldsymbol{q}=0$ if and only if $X \cap Y \neq\{0\}$, for a $k$-dimensional linear subspace $X$ and a $(d+1-k)$-dimensional linear subspace $Y$ with the Plücker coordinates $[\boldsymbol{p}]$ and $[\boldsymbol{q}]$.

For general treatments of these operations, see, e.g., $[1,11]$.

3.2.2. Body-bar frameworks. We shall use the following conventional notation to denote body-bar frameworks and to describe infinitesimal motions. A body-bar framework is a pair $(G, \boldsymbol{q})$, where

- $G=(V, E)$ is a graph;

- $\boldsymbol{q}$ is a mapping called a bar-configuration

$$
\begin{aligned}
\boldsymbol{q}: E & \rightarrow G r(2, W) \subseteq \mathbb{P}\left(\bigwedge^{2} W\right), \\
e & \mapsto\left[\boldsymbol{q}_{e}\right]=\left[q_{e}^{1}, \ldots, q_{e}^{D}\right] .
\end{aligned}
$$

Namely, a line $\boldsymbol{q}(e)$ associated with $e=u v$ represents a bar connecting between two bodies associated with $u$ and $v$. An infinitesimal motion of $(G, \boldsymbol{q})$ is a mapping $\boldsymbol{m}: V \rightarrow \bigwedge^{d-1} W$ satisfying

$$
\boldsymbol{q}_{e} \cdot(\boldsymbol{m}(u)-\boldsymbol{m}(v))=0 \quad \text { for all } e=u v \in E .
$$

This definition is essentially the same as the conventional one used in the bar-joint model, in the sense that it requires the orthogonality of the direction of a bar and the difference of infinitesimal motions assigned to the adjacent bodies. A detailed geometric meaning of (3.3) is explained in Appendix A. (Detailed description can also be found in, e.g., [32, 37, 36, 14].)

The set of infinitesimal motions forms a $D|V|$-dimensional vector space. An infinitesimal motion is called trivial if $\boldsymbol{m}(v)=\boldsymbol{m}(u)$ for all $u, v \in V$. It is easy to see that the collection of trivial motions forms a $D$-dimensional vector space. A body-bar framework is called infinitesimally rigid if every infinitesimal motion is trivial. 
3.2.3. Body-bar matroids. The body-bar matroid $\mathcal{B}(G, \boldsymbol{q})$ is defined as a matroid on $E$ whose rank is the maximum size of independent linear equations in (3.3) (for unknown $\boldsymbol{m}$ ). Namely, $\mathcal{B}(G, \boldsymbol{q})$ is a linear matroid on $E$ in which each edge $e=u v$ is represented by the following vector in $V_{V}\left(=\mathbb{R}^{D|V|}\right)$ :

$$
(0, \cdots, 0, \overbrace{q_{e}^{1}, \ldots, q_{e}^{D}}^{u}, 0, \cdots, 0, \overbrace{-q_{e}^{1}, \ldots,-q_{e}^{D}}^{v}, 0, \cdots, 0) .
$$

Notice that unlike the union of $D$ copies of the graphic matroid, $\left[q_{e}^{1}, \ldots, q_{e}^{D}\right]$ is restricted to $\operatorname{Gr}(2, W)$ for each $e \in E$ (compare to (3.1)). The direct product of this restricted space over all edges is called the bar-configuration space $\mathcal{C}$.

A bar-configuration $\boldsymbol{q}$ is called generic if the rank of every $F \subseteq E$ in $\mathcal{B}(G, \boldsymbol{q})$ is maximized among all bar-configurations. As pointed out in [39], it can be seen that almost all bar-configurations $\boldsymbol{q}$ are generic as follows. Let $B(\boldsymbol{q})$ be the $|E| \times D|V|$ matrix representing $\mathcal{B}(G, \boldsymbol{q})$. Note that the rank of $\mathcal{B}(G, \boldsymbol{q})$ decreases only if a minor of $B(\boldsymbol{q})$ vanishes. Each minor of $B(\boldsymbol{q})$ defines an algebraic variety $S$ of $\mathcal{C}$, which is lower-dimensional than $\mathcal{C}$ since a polynomial generating $S$ is linear with respect to $q_{e}^{1}, \ldots, q_{e}^{D}$ for each $e \in E$. Thus, $\mathcal{C} \backslash S$ is a dense subset of $\mathcal{C}$. Since there are a finite number of minors in $B(\boldsymbol{q})$, the set of points in $\mathcal{C}$ in which no minor vanishes is also a dense subset of $\mathcal{C}$. In other words, almost all bar-configurations are generic.

Notice that once we assume generic bar-configurations, the rank of $\mathcal{B}(G, \boldsymbol{q})$ is determined only by $G$. We hence define the generic body-bar matroid $\mathcal{B}(G)$ as $\mathcal{B}(G, \boldsymbol{q})$ with a (any) generic bar-configuration $\boldsymbol{q}$. The following result is proved by Tay [30]. Simpler proofs based on tree-decompositions are given in $[37,39]$. We shall provide a proof from our viewpoint.

Theorem 3.1 (Tay [30]). Let $G=(V, E)$ be a graph. Then, $\mathcal{B}(G)=D \mathcal{G}(G)$.

Proof. From the discussion given in subsection 3.1,DG(G) is equal to the generic matroid $\mathcal{M}(\mathcal{A})$ associated with the flat family $\mathcal{A}=\left\{A_{e}: e \in E\right\}$ defined by

$$
A_{e}=\left\{[0, \cdots, 0, \stackrel{u}{\boldsymbol{\alpha}}, 0, \cdots, 0, \stackrel{v}{-}, \boldsymbol{\alpha}, 0, \cdots, 0]:[\boldsymbol{\alpha}] \in \mathbb{P}^{D-1}\right\} \subseteq \mathbb{P}\left(V_{V}\right) .
$$

In order to prove $\mathcal{B}(G)=\mathcal{M}(\mathcal{A})$, it is sufficient to show that the representative point $x_{e}$ of $A_{e}$ (that defines $\mathcal{M}(\mathcal{A})$ ) can be taken to be in general position from

$$
\hat{A}_{e}=\left\{\left[0, \cdots, 0, \stackrel{u}{\boldsymbol{\alpha}}, 0, \cdots, 0,-{ }^{v} \boldsymbol{\alpha}, 0, \cdots, 0\right]:[\boldsymbol{\alpha}] \in G r(2, W)\right\} \subseteq \mathbb{P}\left(V_{V}\right) .
$$

Specifically, we need to show that there exists $X=\left\{x_{e} \in \hat{A}_{e}: e \in E\right\}$ such that, for each $X^{\prime} \subseteq X$ and $x_{e} \in X^{\prime}$,

$$
x_{e} \in \overline{X^{\prime}-x_{e}} \Rightarrow A_{e} \subseteq \overline{X^{\prime}-x_{e}}
$$

(c.f. (2.4)). Let us consider the case $d=3$ (and $D=6$ ). For $e=u v \in E$, let us pick a point

$$
x_{e}=[0, \cdots, 0, \overbrace{x_{e}^{1}, \ldots, x_{e}^{6}}^{u}, 0, \cdots, 0, \overbrace{-x_{e}^{1}, \ldots,-x_{e}^{6}}^{v}, 0, \cdots, 0] \in A_{e} .
$$

Then, $x_{e} \in \hat{A}_{e}$ if and only if $x_{e}^{1} x_{e}^{6}-x_{e}^{2} x_{e}^{5}+x_{e}^{3} x_{e}^{4}=0$. We now focus on a fivedimensional affine space $\mathbb{A}$ by setting $x_{e}^{4}=1$. Note that $\operatorname{Gr}(2, W) \cap \mathbb{A}$ is a smooth four-dimensional manifold parameterized by $x_{e}^{1}, x_{e}^{2}, x_{e}^{5}, x_{e}^{6}$ since $x_{e}^{3}=-x_{e}^{1} x_{e}^{6}+x_{e}^{2} x_{e}^{5}$. 

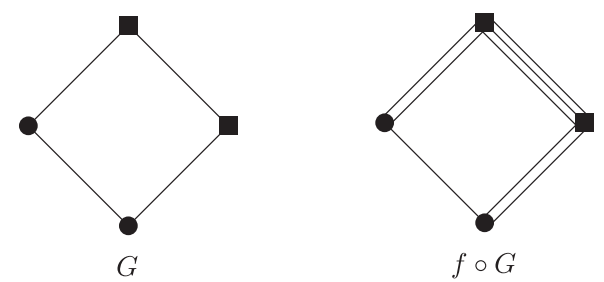

FIG. 4.1. Example of $f \circ G$ for $D=3$, where circles and squares represent vertices of $R$ and $B$, respectively.

Let us take $x_{e}$ so that the set of parameters $x_{e}^{1}, x_{e}^{2}, x_{e}^{5}, x_{e}^{6}$ for all $e \in E$ is algebraically independent over $\mathbb{Q}$. Suppose, for a contradiction, that $x_{e} \in \overline{X^{\prime}-x_{e}}$ but $A_{e} \not \subset \overline{X^{\prime}-x_{e}}$ for some $e=u v$. Let us consider a hyperplane $H$ of $\mathbb{P}\left(V_{V}\right)$ that contains $\overline{X^{\prime}-x_{e}}$ but does not contain $A_{e}$. We can take such a hyperplane $H$ so that each coefficient is written as a polynomial of $\left\{x_{e^{\prime}}^{1}, x_{e^{\prime}}^{2}, x_{e^{\prime}}^{5}, x_{e^{\prime}}^{6}: e^{\prime} \in E-e\right\}$ over $\mathbb{Q}$. Moreover, $H \cap \hat{A}_{e}$ is a lower-dimensional subspace of $\hat{A}_{e}$ since $\operatorname{Gr}(2, W)$ is quadratic and irreducible. In particular, $H$ does not contain $\hat{A}_{e}$. Therefore, if $x_{e} \in H$, then $\left\{x_{e}^{1}, x_{e}^{2}, x_{e}^{5}, x_{e}^{6}: e \in E\right\}$ satisfies a nontrivial algebraic relation over $\mathbb{Q}$, contradicting the choice of $x_{e}$.

The general $d$-dimensional case follows in the same way based on the following fact. If $\operatorname{Gr}(2, W)$ is restricted to a $(D-1)$-dimensional affine space $\mathbb{A}$ by fixing one coordinate, then $\operatorname{Gr}(2, W) \cap \mathbb{A}$ is known to be a smooth $2(d-1)$-dimensional manifold (see, e.g., [10]). Moreover, each coordinate of a point in $\operatorname{Gr}(2, W) \cap \mathbb{A}$ is written as a rational function of $2(d-1)$ parameters with coefficients in $\mathbb{Q}$. Thus, we can apply the exact same argument.

4. Body-rod-bar frameworks. We now provide our main result on the generic rigidity of body-rod-bar frameworks. We first introduce a counting matroid defined on graphs in subsection 4.1, and then in subsection 4.2 we show that generic rigidity of body-rod-bar frameworks can be characterized by the combinatorial matroid.

\subsection{Combinatorial truncated matroids.}

4.1.1. Count matroids. Let $G=(V, E)$ be a graph with an (ordered) partition $\mathcal{P}=\{B, R\}$ of $V$ into two subsets (where $B$ and $R$ will represent a set of bodies and a set of rods, respectively, in the next subsection). We define an integer-valued function $f$ on $E$ defined by

$$
f(F)=D(|V(F)|-1)-|R(F)| \quad(F \subseteq E),
$$

where $R(F)$ denotes the set of vertices in $R$ spanned by $F$, and $D=\left(\begin{array}{c}d+1 \\ 2\end{array}\right)$ as in section 3. Then, $f$ is a monotone submodular function on $E$, since $f(F)=D|B(F)|+$ $(D-1)|R(F)|-D$ and $|B(\cdot)|$ and $|R(\cdot)|$ are both monotone and submodular. Thus, $f$ induces the matroid $\left(E, r_{f}\right)$ on $E$, denoted $\mathcal{M}_{f}(G, \mathcal{P})$. If the bipartition $\mathcal{P}$ is clear from the context, we abbreviate it and simply denote $\mathcal{M}_{f}(G)$. This matroid is a special case of so-called count matroids on undirected graphs; see, e.g., [4, section 13.5] for more detail.

We denote by $f \circ G$ the graph obtained from $G$ by replacing each edge $e$ by $f(e)$ parallel copies of $e$ (see Figure 4.1). Also, $f \circ e$ denotes the set of corresponding copies of $e$, and let $f \circ F=\bigcup_{e \in F} f \circ e$. We can naturally extend $f$ to that on $f \circ E$ by setting $f(F)=D|V(F)|-D-|R(F)|$ for $F \subseteq f \circ E$. 
Let us consider $\hat{f}: 2^{E} \rightarrow \mathbb{Z}$ defined by (2.1), i.e., for $F \subseteq E$,

$$
\hat{f}(F)=\min \left\{\sum_{i=1}^{k}\left(D\left(\left|V\left(F_{i}\right)\right|-1\right)-\left|R\left(F_{i}\right)\right|\right): \text { a partition }\left\{F_{1}, \ldots, F_{k}\right\} \text { of } F\right\} \text {. }
$$

As mentioned in subsection 2.1, $\hat{f}$ is a monotone submodular function satisfying $f(\emptyset)=0$, and thus $(E, \hat{f})$ forms a polymatroid, denoted by $\mathcal{P} \mathcal{M}_{f}(G, \mathcal{P})$ (or simply by $\left.\mathcal{P} \mathcal{M}_{f}(G)\right)$. The following lemma implies that $\mathcal{P} \mathcal{M}_{f}(G)$ is essentially the same as $\mathcal{M}_{f}(f \circ G)$.

Lemma 4.1. For any $F \subseteq E, \hat{f}(F)=r_{f}(f \circ F)$. Namely, the rank of $F \subseteq E$ in $\mathcal{P} \mathcal{M}_{f}(G)$ is equal to the rank of $f \circ F$ in $\mathcal{M}_{f}(f \circ G)$.

Proof. Recall that for any $F \subseteq E, r_{f}(f \circ F)$ is written as $r_{f}(f \circ F)=\min \left\{\left|F_{0}\right|+\right.$ $\left.\sum_{i=1}^{k} f\left(F_{i}\right)\right\}$, where the minimum is taken over partitions $\left\{F_{0}, F_{1}, \ldots, F_{k}\right\}$ of $f \circ F$ such that $F_{1}, \ldots, F_{k} \neq \emptyset($ see $(2.3))$. Let $\left\{F_{0}^{*}, F_{1}^{*}, \ldots, F_{k}^{*}\right\}$ be a partition of $f \circ F$ that attains that minimum. Since $|f \circ e|=f(e)$ for every $e \in E$, we may assume $F_{0}^{*}=\emptyset$. Also, since $f(f \circ F)=f(F)$ for any $F \subseteq E$, we may assume that each $F_{i}^{*}(\subseteq f \circ F)$ is written as $F_{i}^{*}=f \circ F_{i}^{\prime}$ for some $F_{i}^{\prime} \subseteq F$. Thus, $r_{f}(f \circ F)$ is actually written as $r_{f}(f \circ F)=\min \left\{\sum_{i=1}^{k} f\left(f \circ F_{i}^{\prime}\right)\right\}=\min \left\{\sum_{i=1}^{k} f\left(F_{i}^{\prime}\right)\right\}$, where the minimum is taken over all partitions $\left\{F_{1}^{\prime}, \ldots, F_{k}^{\prime}\right\}$ of $F$. This is exactly the definition of $\hat{f}(F)$.

A reduction technique of general polymatroids to matroids can be found in, e.g., [28, section 44.6b].

4.1.2. Properties of $\mathcal{M}_{\boldsymbol{f}}$. We now show several properties of $\mathcal{M}_{f}(G, \mathcal{P})$ for a graph $G=(V, E)$ with a bipartition $\mathcal{P}=\{B, R\}$ of $V$. (These lemmas are generally known for count matroids. We provide proofs for completeness.)

Lemma 4.2. Let $C$ be a circuit of $\mathcal{M}_{f}(G)$. Then, $r_{f}(C)=f(C)$.

Proof. Since $C$ is a minimal dependent set, $|C|>f(C)$ and $|C|-1=|C-e| \leq$ $f(C-e) \leq f(C)$ for any $e \in C$. This implies $|C|=f(C)+1$. Thus, $r_{f}(C)=|C|-1=$ $f(C)$.

Lemma 4.3. Let $F \subseteq E$ be a nontrivial $M$-connected set in $\mathcal{M}_{f}(G)$. Then, $r_{f}(F)=f(F)$.

Proof. Suppose $r_{f}(F)<f(F)$. Then, there are $u, v \in V(F)$ with $u v \notin F$ such that $r_{f}(F+u v)=r_{f}(F)+1$. Let us take two distinct edges $e$ and $e^{\prime}$ of $F$ incident to $u$ and $v$, respectively. (It is easy to see that two such edges exist since $F$ is $M$-connected.) Since $F$ is $M$-connected, there is a circuit $C \subseteq F$ that contains $e$ and $e^{\prime}$. Then, by Lemma 4.2 and by $f(C+u v)=f(C)$, we obtain $r_{f}(C+u v) \leq f(C+u v)=f(C)=r_{f}(C)$, implying $r_{f}(C+u v)=r_{f}(C)$. In other words, $u v$ is contained in the closure of $C$. This contradicts $r_{f}(F+u v)=r_{f}(F)+1$.

Lemma 4.4. Let $F \subseteq E$ be a nontrivial $M$-connected set in $\mathcal{M}_{f}(G)$. Then, the closure of $F$, that is, $\left\{e \in E(G): r_{f}(F+e)=r_{f}(F)\right\}$, is the set of edges induced by $V(F)$. In particular, if $F$ is an $M$-connected component, then $(V(F), F)$ is an induced subgraph.

Proof. Since $f(F+e)=f(F)$ holds for any edge $e$ induced by $V(F)$, the claim follows from Lemma 4.3.

4.1.3. Properties of $\mathcal{P} \mathcal{M}_{\boldsymbol{f}}$. Let us consider $\mathcal{M}_{f}(f \circ G)$ for a graph $G=(V, E)$ with a bipartition $\mathcal{P}$. By Lemma 4.4, an $M$-connected component $C$ of $\mathcal{M}_{f}(f \circ G)$ is either trivial or of the form $C=f \circ F$ for some $F \subseteq E$ with $|F| \geq 2$. The $M$ connected component decomposition of $\mathcal{M}_{f}(f \circ G)$ thus induces a unique partition $\left\{C_{1}, \ldots, C_{k}\right\}$ of $E$ such that $C_{i}$ is singleton or $f \circ C_{i}$ is an $M$-connected component in $\mathcal{M}_{f}(f \circ G)$. The following lemma says that this partition coincides with the $P$ connected component decomposition of $\mathcal{P} \mathcal{M}_{f}(G)$. 


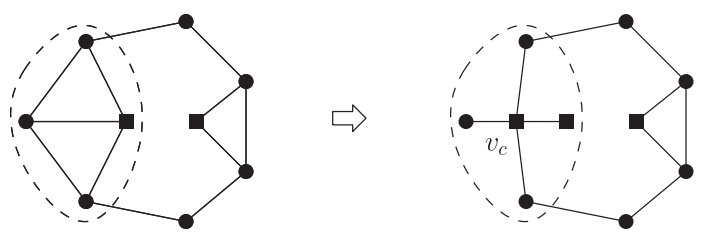

Fig. 4.2. Simplification.

Lemma 4.5. For a graph $G=(V, E)$ with a bipartition $\mathcal{P}=\{B, R\}$ of $V$, the following hold:

(i) Any nontrivial $M$-connected component $X$ of $\mathcal{M}_{f}(f \circ G)$ can be written as $X=f \circ F$ for some nontrivial $P$-connected component $F \subseteq E$.

(ii) If $F \subseteq E$ is a nontrivial $P$-connected set in $\mathcal{P} \mathcal{M}_{f}(G)$, then $f \circ F$ is $M$ connected in $\mathcal{M}_{f}(G)$.

(iii) The P-connected component decomposition $\left\{C_{1}, \ldots, C_{k}\right\}$ of $\mathcal{P} \mathcal{M}_{f}(G)$ is a minimizer of the right-hand side of (4.2).

Proof. (i) and (ii) are direct consequences of Lemma 4.1.

For the last claim, Lemma 4.1, Lemma 4.4, and (ii) imply $\hat{f}(E)=\sum_{i=1}^{k} \hat{f}\left(C_{i}\right)=$ $\sum_{i=1}^{k} r_{f}\left(f \circ C_{i}\right)=\sum_{i=1}^{k} f\left(f \circ C_{i}\right)=\sum_{i=1}^{k} f\left(C_{i}\right)$.

For a simple graph $G=(V, E)$, it is sometimes useful to introduce the underlying complete simple graph $K(V)$ on $V$ that contains $G$ and extend $\mathcal{P} \mathcal{M}_{f}(G, \mathcal{P})$ to $\mathcal{P} \mathcal{M}_{f}(K(V), \mathcal{P})$. We shall denote by cl the closure operator of $\mathcal{P} \mathcal{M}_{f}(K(V), \mathcal{P})$, i.e., $\operatorname{cl}(F)=\{u v \in K(V): \hat{f}(F+u v)=\hat{f}(F)\}$ for $F \subseteq E$. Then, by Lemma 4.5, $\operatorname{cl}(F)$ forms the complete graph on $V(F)$ if $F$ is $P$-connected.

The following lemmas are key observations used in the proof of the main theorem (Theorem 4.12).

Lemma 4.6. Let $G=(V, E)$ be a connected simple graph with a bipartition $\mathcal{P}=\{B, R\}$ of $V$. Suppose $D \geq 6$. Then $G$ has (i) three vertices each of which is spanned by exactly two $P$-connected components of $\mathcal{P} \mathcal{M}_{f}(G)$ or (ii) a vertex that is spanned by only one P-connected component.

Proof. Let $\left\{C_{1}, \ldots, C_{k}\right\}$ be the $P$-connected component decomposition of $\mathcal{P} \mathcal{M}_{f}(G)$. Note then, since $G$ is simple, any nontrivial $P$-connected component $C_{i}$ satisfies $\left|V\left(C_{i}\right)\right| \geq 3$.

For each nontrivial $C_{i}$, we consider the following graph operation on $G$, called the simplification of $C_{i}$; remove $C_{i}$, insert a new vertex $v_{c}$ to $B$, and connect each vertex of $V\left(C_{i}\right)$ with $v_{c}$. Namely, we replace the induced subgraph $\left(V\left(C_{i}\right), C_{i}\right)$ by the star $\left(V\left(C_{i}\right) \cup\left\{v_{c}\right\}, S\right)$ with the centered new vertex $v_{c}$ and the set $S$ of edges between $v_{c}$ and $V\left(C_{i}\right)$ (see Figure 4.2).

Claim 4.7. Let $C$ be a nontrivial P-connected component of $\mathcal{P} \mathcal{M}_{f}(G, \mathcal{P})$. Let $G^{\prime}$ be the graph obtained by the simplification of $C$, where we denote $V\left(G^{\prime}\right)=V \cup\left\{v_{c}\right\}$ and $E\left(G^{\prime}\right)=(E \backslash C) \cup S$, with the bipartition $\mathcal{P}^{\prime}=\left\{B \cup\left\{v_{c}\right\}, R\right\}$ of $V\left(G^{\prime}\right)$. Then, each new edge $e \in f \circ S$ is a coloop in $\mathcal{M}_{f}\left(f \circ G^{\prime}, \mathcal{P}^{\prime}\right)$.

Proof. From the definition of $f$, it is easy to check that $f \circ S$ is independent in $\mathcal{M}_{f}\left(f \circ G^{\prime}, \mathcal{P}^{\prime}\right)$. Since $C$ is a $P$-connected component, we have $\operatorname{cl}(S) \cap \operatorname{cl}\left(C^{\prime}\right)=\emptyset$ for any other $P$-connected component $C^{\prime}$ of $\mathcal{P} \mathcal{M}_{f}(G, \mathcal{P})$. This implies that there is no circuit of $\mathcal{M}_{f}\left(f \circ G^{\prime}, \mathcal{P}^{\prime}\right)$ that intersects both $f \circ(E \backslash C)$ and $f \circ S$. Since $f \circ S$ is independent, there is also no circuit within $f \circ S$ and thus no circuit that contains $e \in f \circ S$ in $\mathcal{M}_{f}\left(f \circ G^{\prime}, \mathcal{P}^{\prime}\right)$. 
Claim 4.7 implies that if we apply the simplification of the $P$-connected component $C_{i}$, then no new nontrivial $P$-connected component appears, and $C_{1}, \ldots, C_{i-1}$, $C_{i+1}, \ldots, C_{k}$ are all nontrivial $P$-connected components in the resulting polymatroid. Hence, we may apply the simplifications for all $C_{1}, \ldots, C_{k}$ simultaneously. Let $G^{\prime \prime}$ be the resulting graph with the corresponding bipartition $\mathcal{P}^{\prime \prime}$ of $V\left(G^{\prime \prime}\right)$ after the simplifications. Notice that the degree of each vertex $v \in V(G)$ in $G^{\prime \prime}$ corresponds to the number of $P$-connected components among $C_{1}, \ldots, C_{k}$ that span $v$ in $G$. We also remark that each vertex of $V\left(G^{\prime \prime}\right) \backslash V(G)$ has degree at least three since $\left|V\left(C_{i}\right)\right| \geq 3$. Thus, to complete the proof, it is sufficient to show that $G^{\prime \prime}$ has at least three vertices of degree 2 or a vertex of degree 1 . To see this, observe that $f \circ E\left(G^{\prime \prime}\right)$ is independent in $\mathcal{M}_{f}\left(f \circ G^{\prime \prime}, \mathcal{P}^{\prime \prime}\right)$ by Claim 4.7. So, we have $\left|f \circ E\left(G^{\prime \prime}\right)\right|=r_{f}\left(f \circ E\left(G^{\prime \prime}\right)\right)$. This implies $(D-2)\left|E\left(G^{\prime \prime}\right)\right| \leq \sum_{e \in E\left(G^{\prime \prime}\right)} f(e)=\left|f \circ E\left(G^{\prime \prime}\right)\right|=r_{f}\left(f \circ E\left(G^{\prime \prime}\right)\right) \leq D\left|V\left(G^{\prime \prime}\right)\right|-D$. Let $d_{\text {avg }}$ be the average degree of $G^{\prime \prime}$. Then we have

$$
d_{\mathrm{avg}}=\frac{2\left|E\left(G^{\prime \prime}\right)\right|}{\left|V\left(G^{\prime \prime}\right)\right|} \leq \frac{2 D}{D-2}\left(1-\frac{1}{\left|V\left(G^{\prime \prime}\right)\right|}\right) .
$$

Suppose there is no vertex of degree 1. Denoting the set of vertices of degree 2 in $G^{\prime \prime}$ by $V_{2}$, we have

$$
d_{\text {avg }} \geq 3-\frac{\left|V_{2}\right|}{\left|V\left(G^{\prime \prime}\right)\right|} .
$$

Putting them together, we obtain

$$
\left|V_{2}\right| \geq \frac{2 D}{D-2}+\frac{D-6}{D-2}\left|V\left(G^{\prime \prime}\right)\right| \geq \frac{2 D}{D-2}+\frac{D-6}{D-2}=3
$$

(where we used $D \geq 6$ and $\left|V\left(G^{\prime \prime}\right)\right| \geq 1$ ). This completes the proof.

Remark. Lemma 4.6 does not hold for $d=2$ and $D=3$. For example, in the cube graph, all $P$-connected components are trivial and hence each vertex is spanned by three $P$-connected components since each vertex has degree 3 .

LEMma 4.8. Let $G=(V, E)$ be a simple graph for which $E$ is $P$-connected in $\mathcal{P} \mathcal{M}_{f}(G, \mathcal{P})$. Suppose further that there are two disjoint nonempty $P$-connected sets $C_{1}$ and $C_{2}$ both of which span a vertex $u \in V$. Then, $G$ contains a $P$-connected set $C$ such that $C_{1} \subseteq C \subseteq E \backslash C_{2}$ and $u v \in \operatorname{cl}(C) \cap \operatorname{cl}(E \backslash C)$ for some uv $\in K(V)$.

Proof. Let us take an inclusion-wise maximal $P$-connected set $C$ such that $C_{1} \subseteq$ $C \subseteq E \backslash C_{2}$. Since $E$ is $P$-connected, we have $\operatorname{cl}(C) \cap \operatorname{cl}(E \backslash C) \neq \emptyset$, and hence there is an edge $v w \in K(V)$ such that $v w \in \operatorname{cl}(C) \cap \operatorname{cl}(E \backslash C)$. If either $v=u$ or $w=u$, then $C$ satisfies the required property. Thus, suppose to the contrary that every edge in $\operatorname{cl}(C) \cap \operatorname{cl}(E \backslash C)$ is not incident to $u$. Let $C^{\prime}$ be a $P$-connected set in $E \backslash C$ with $v w \in \operatorname{cl}\left(C^{\prime}\right)$. Since $v w \in \operatorname{cl}(E \backslash C)$, such $C^{\prime}$ exist. $\left(C^{\prime}=\{v w\}\right.$ may hold if $v w \in E \backslash C$.)

If $C_{2} \cap C^{\prime} \neq \emptyset$, then $C_{2} \cup C^{\prime}$ is $P$-connected, and hence $\operatorname{cl}\left(C_{2} \cup C^{\prime}\right)$ forms the complete graph on $V\left(C_{2} \cup C^{\prime}\right)$. Since $u \in V\left(C_{2}\right)$ and $v \in V\left(C^{\prime}\right)$, we obtain $u v \in$ $\operatorname{cl}\left(C_{2} \cup C^{\prime}\right) \subseteq \operatorname{cl}(E \backslash C)$. On the other hand, since $C$ is $P$-connected with $u, v \in V(C)$, we also have $u v \in \operatorname{cl}(C)$. This, however, contradicts that every edge in $\operatorname{cl}(C) \cap \operatorname{cl}(E \backslash C)$ is not incident to $u$

If $C_{2} \cap C^{\prime}=\emptyset$, then $C \cup C^{\prime}$ is $P$-connected since $\operatorname{cl}(C) \cap \operatorname{cl}\left(C^{\prime}\right)$ is nonempty, and thus $C \cup C^{\prime}$ is $P$-connected with $C_{1} \subseteq C \cup C^{\prime} \subseteq E \backslash C_{2}$ and is larger than $C$, contradicting the choice of $C$.

\subsection{Generic body-rod-bar matroids.}

4.2.1. Body-rod-bar frameworks. A body-rod-bar framework is a body-bar framework in which some of bodies are degenerate as one-dimensional bodies in the case of $d=3$. In the general dimensional case, a body-rod-bar framework can be 
defined as a structure consisting of $d$-dimensional subspaces (bodies) and $(d-2)$ dimensional flats (rods) mutually linked by one-dimensional lines (bars). (The term "rod" is actually appropriate only for $d=3$.) We thus define a body-rod-bar framework as $(G, \boldsymbol{q}, \boldsymbol{r})$, where

- $G=(V, E)$ is a graph with a bipartition $\mathcal{P}=\{B, R\}$ of $V$;

- $r$ is a mapping called a rod-configuration,

$$
\begin{aligned}
\boldsymbol{r}: R & \rightarrow G r(d-1, W) \subseteq \mathbb{P}\left(\bigwedge^{d-1} W\right), \\
v & \mapsto\left[\boldsymbol{r}_{v}\right]=\left[r_{v}^{1}, \ldots, r_{v}^{D}\right] ;
\end{aligned}
$$

- $\boldsymbol{q}$ is a bar-configuration,

$$
\begin{aligned}
\boldsymbol{q}: R & \rightarrow G r(2, W) \subseteq \mathbb{P}\left(\bigwedge^{2} W\right), \\
e & \mapsto\left[\boldsymbol{q}_{e}\right]=\left[q_{e}^{1}, \ldots, q_{e}^{D}\right],
\end{aligned}
$$

satisfying the incidence condition:

$$
\boldsymbol{q}_{e} \cdot \boldsymbol{r}_{v}=0 \quad \text { if } e \in E \text { is incident to } v \in R .
$$

That is, $\boldsymbol{r}(v)$ represents a rod associated with $v \in R$, and $\left[\boldsymbol{r}_{v}\right]$ denotes the Plücker coordinate of the rod. Recall that for $[\boldsymbol{q}] \in G r(2, W)$ and $[\boldsymbol{r}] \in G r(d-1, W), \boldsymbol{q} \cdot \boldsymbol{r}=0$ holds if and only if the corresponding linear subspaces have a nonzero intersection (equivalently, the corresponding flats have a nonempty intersection). Thus, the system (4.3) describes incidence constraints between rods and bars. Throughout the subsequent discussions, we also impose an additional condition that all rods are distinct, i.e., $\boldsymbol{r}(u) \neq \boldsymbol{r}(v)$ for any $u, v \in R$ with $u \neq v$.

As in the case of body-bar frameworks, an infinitesimal motion of $(G, \boldsymbol{q}, \boldsymbol{r})$ is defined as $\boldsymbol{m}: V \rightarrow \bigwedge^{d-1} W$ satisfying (3.3), and $\boldsymbol{m}$ is called trivial if $\boldsymbol{m}(u)=\boldsymbol{m}(v)$ for all $u, v \in V$.

For each $v \in R$, define $\boldsymbol{m}_{v}: V \rightarrow \bigwedge^{d-1} W$ by $\boldsymbol{m}_{v}(v)=\boldsymbol{r}_{v}$ and $\boldsymbol{m}_{v}(u)=0$ for $u \in V \backslash\{v\}$. Then, by incidence condition (4.3), $\boldsymbol{m}_{v}$ always satisfies (3.3), and $\boldsymbol{m}_{v}$ is an infinitesimal motion of $(G, \boldsymbol{q}, \boldsymbol{r})$. Conventionally, we also include $\boldsymbol{m}_{v}$ in the set of trivial motions. The set of all trivial motions thus forms a $(D+|R|)$-dimensional vector space. If every motion of $(G, \boldsymbol{q}, \boldsymbol{r})$ is trivial, it is said to be infinitesimally rigid.

4.2.2. Body-rod-bar matroids. As defined in the body-bar matroid, the bodyrod-bar matroid $\mathcal{B R}(G, \boldsymbol{q}, \boldsymbol{r})$ is defined as that on $E$ whose rank is the maximum size of independent linear equations in (3.3) (for unknown $\boldsymbol{m}$ ). From the definition, $(G, \boldsymbol{q}, \boldsymbol{r}$ ) is infinitesimally rigid if and only if the rank of $\mathcal{B R}(G, \boldsymbol{q}, \boldsymbol{r})$ is $D|V|-(D+|R|)$. The following theorem is our main result.

THEOREM 4.9. Let $G=(V, E)$ be a graph with a bipartition $\mathcal{P}=\{B, R\}$ of $V$ and $f$ be the function defined by (4.1). Suppose $d \geq 3$. Then, for almost all bar-configurations $\boldsymbol{q}$ and almost all rod-configurations $\boldsymbol{r}, \mathcal{B R}(G, \boldsymbol{q}, \boldsymbol{r})=\mathcal{M}_{f}(G, \mathcal{P})$. Namely, $I \subseteq E$ is independent in $\mathcal{B R}(G, \boldsymbol{q}, \boldsymbol{r})$ if and only if $|F| \leq D|V(F)|-D-$ $|R(F)|$ for any nonempty $F \subseteq I$.

We need to introduce a notation for the proof. Let $\boldsymbol{r}: R \rightarrow G r(d-1, W)$ be a rod-configuration. For each $v \in R$, let $H_{\boldsymbol{r}}(v)$ be the dual hyperplane to the point $\left[\boldsymbol{r}_{v}\right]$ in $\mathbb{P}\left(\bigwedge^{2} W\right)$, i.e., $H_{\boldsymbol{r}}(v)=\left\{[\boldsymbol{p}] \in \mathbb{P}\left(\bigwedge^{2} W\right): \boldsymbol{p} \cdot \boldsymbol{r}_{v}=0\right\}$. For easie of the description, we also define $H_{\boldsymbol{r}}(v)$ for $v \in B$ to be $H_{\boldsymbol{r}}(v)=\mathbb{P}\left(\bigwedge^{2} W\right)$. Notice that, due to the incidence condition (4.3), the space of $\boldsymbol{q}_{u v}$ is restricted to $G r(2, W) \cap H_{\boldsymbol{r}}(u) \cap H_{\boldsymbol{r}}(v)$ 
for $u v \in E$. We hence define two subspaces associated with $e=u v \in E$ as follows:

$$
\begin{aligned}
& A_{e}(\boldsymbol{r})=\left\{\left[0, \cdots, 0, \boldsymbol{\alpha}, 0, \cdots, 0,-{ }^{v} \boldsymbol{\alpha}, 0, \cdots, 0\right]:[\boldsymbol{\alpha}] \in \mathbb{P}\left(\bigwedge^{2} W\right) \cap H_{\boldsymbol{r}}(u) \cap H_{\boldsymbol{r}}(v)\right\} \\
& \hat{A}_{e}(\boldsymbol{r})=\left\{[0, \cdots, 0, \boldsymbol{\alpha}, 0, \cdots, 0,-\boldsymbol{\alpha}, 0, \cdots, 0]:[\boldsymbol{\alpha}] \in G r(2, W) \cap H_{\boldsymbol{r}}(u) \cap H_{\boldsymbol{r}}(v)\right\} .
\end{aligned}
$$

Also, let $\mathcal{A}(\boldsymbol{r})=\left\{A_{e}(\boldsymbol{r}): e \in E\right\}$, and as before let $\mathcal{A}_{F}(\boldsymbol{r})=\left\{A_{e}(\boldsymbol{r}): e \in F\right\}$ for $F \subseteq E$.

The proof of Theorem 4.9 proceeds as follows. We first show that $\mathcal{B R}(G, \boldsymbol{q}, \boldsymbol{r})$ is equal to the linear matroid $\mathcal{M}(\mathcal{A}(\boldsymbol{r}))$ associated with the flat family $\mathcal{A}(\boldsymbol{r})$ for almost all configurations (Theorem 4.10). We then provide an explicit formula of the rank of $\mathcal{A}(\boldsymbol{r})$ in terms of the underlying graph $G$ (Theorem 4.12) and finally show that $\mathcal{M}(\mathcal{A}(\boldsymbol{r}))$ is indeed equal to $\mathcal{M}_{f}(G, \mathcal{P})$ (Corollary 4.13).

THEOREM 4.10. Let $G=(V, E)$ be a graph with a bipartition $\mathcal{P}=\{B, R\}$. Then, for almost all rod-configurations $\boldsymbol{r}$ and bar-configurations $\boldsymbol{q}, \mathcal{B R}(G, \boldsymbol{q}, \boldsymbol{r})=$ $\mathcal{M}(\mathcal{A}(\boldsymbol{r}))$.

Proof. The proof is basically the same as that of Theorem 3.1. Recall that $\mathcal{B R}(G, \boldsymbol{q}, \boldsymbol{r})$ is a linear matroid on $E$ in which each element $e=u v \in E$ is represented by

$$
\left(0, \cdots, 0, \boldsymbol{q}_{e}, 0, \cdots, 0,-{ }^{v} \boldsymbol{q}_{e}, 0, \cdots, 0\right),
$$

where $\left[\boldsymbol{q}_{e}\right]$ is restricted to $G r(2, W) \cap H_{\boldsymbol{r}}(u) \cap H_{\boldsymbol{r}}(v)$ in the case of body-rod-bar frameworks. Hence, to prove $\mathcal{B R}(G, \boldsymbol{q}, \boldsymbol{r})=\mathcal{M}(\mathcal{A}(\boldsymbol{r}))$, it is sufficient to show that a representative point $x_{e}=\left[0, \ldots, 0, x_{e}^{1}, \ldots, x_{e}^{D}, 0, \ldots, 0,-x_{e}^{1}, \ldots,-x_{e}^{D}, 0, \ldots, 0\right]$ of $A_{e}(\boldsymbol{r})$ can be taken from $\hat{A}_{e}(\boldsymbol{r})$ so that $X=\left\{x_{e}: e \in E\right\}$ is in generic position (in the sense of definition (2.4)).

Let us consider the case $d=3$. Let us take $\boldsymbol{r}$ so that $\boldsymbol{r}(u) \neq \boldsymbol{r}(v)$ for each $u, v \in V$ with $u \neq v$. Then, for each $e=u v \in E, A_{e}(\boldsymbol{r})$ is isomorphic to $\mathbb{P}\left(\bigwedge^{2} W\right) \cap H_{\boldsymbol{r}}(u) \cap$ $H_{\boldsymbol{r}}(v)=\mathbb{P}^{k}$, where $k=3$ if $u, v \in R ; k=4$ if either $u \in R$ or $v \in R$; otherwise $k=5$. Recall that the quadratic variety $\operatorname{Gr}(2, W) \cap H_{\boldsymbol{r}}(u) \cap H_{\boldsymbol{r}}(v)$ is singular if the associated matrix is singular. Since the determinant of the associated matrix is a polynomial of entries of $\boldsymbol{r}(u)$ and $\boldsymbol{r}(v), G r(2, W) \cap H_{\boldsymbol{r}}(u) \cap H_{\boldsymbol{r}}(v)$ becomes a nonsingular quadratic variety of $\mathbb{P}^{k}$ for almost all rod-configurations $\boldsymbol{r}$. Then, by setting $x_{e}^{4}=1$, it can be easily checked that $\operatorname{Gr}(2, W) \cap H_{\boldsymbol{r}}(u) \cap H_{\boldsymbol{r}}(v)$ can be parameterized by $x_{e}^{1}$ and $x_{e}^{2}$ such that the rest of coordinates $x_{e}^{3}, \ldots, x_{e}^{6}$ are described as rational functions of $x_{e}^{1}$ and $x_{e}^{2}$ with coefficients in $\mathbb{Q}$. If we take $x_{e}$ so that $\left\{x_{e}^{1}, x_{e}^{2}: e \in E\right\}$ is algebraically independent over $\mathbb{Q}, X=\left\{x_{e}: e \in E\right\}$ is in generic position by the same reason as the proof of Theorem 3.1.

The general $d$-dimensional case follows in the same way, as each coordinate of a point in $\operatorname{Gr}(2, W) \cap \mathbb{A}$ is written as a rational function of $2(d-1)$ parameters among $x_{e}^{i, j}(1 \leq i<j \leq d+1)$, if $\operatorname{Gr}(2, W)$ is restricted to a $(D-1)$-dimensional affine space $\mathbb{A}$ (see, e.g., $[10])$.

As noted above, $\mathcal{B R}(G, \boldsymbol{q}, \boldsymbol{r})$ has rank at most $D|V|-D-|R|$ since the corresponding framework $(G, \boldsymbol{q}, \boldsymbol{r})$ always has $D+|R|$ trivial motions. The same argument can be applied to show the following fact.

Lemma 4.11. Let $G=(V, E)$ be a graph with a bipartition $\mathcal{P}=\{B, R\}$ of $V$. Then, for any rod-configuration $\boldsymbol{r}$ such that $\boldsymbol{r}(u) \neq \boldsymbol{r}(v)$ for $u, v \in R$ with $u \neq v$, $\operatorname{rank}(\overline{\mathcal{A}(\boldsymbol{r})}) \leq D|V|-D-|R|$.

The following is a key result for proving Theorem 4.9. 
TheOREM 4.12. Let $G=(V, E)$ be a graph with a bipartition $\mathcal{P}=\{B, R\}$ of $V$. If $d \geq 3$, then for almost all rod-configurations $\boldsymbol{r}$,

$$
\operatorname{rank}(\overline{\mathcal{A}(\boldsymbol{r})})=\min \left\{\sum_{i=1}^{k}\left(D\left|V\left(E_{i}\right)\right|-D-\left|R\left(E_{i}\right)\right|\right)\right\},
$$

where the minimum is taken over all partitions $\left\{E_{1}, \ldots, E_{k}\right\}$ of $E$ into nonempty subsets. Namely, the linear polymatroid $\mathcal{P} \mathcal{M}(\mathcal{A}(\boldsymbol{r}))$ defined by $\mathcal{A}(\boldsymbol{r})$ is equal to the combinatorial polymatroid $\mathcal{P} \mathcal{M}_{f}(G, \mathcal{P})$ for almost all rod-configurations $\boldsymbol{r}$.

One direction of Theorem 4.12 is straightforward from Lemma 4.11. For any partition $\left\{E_{1}, \ldots, E_{k}\right\}$ of $E$, we have $\operatorname{rank}(\overline{\mathcal{A}(\boldsymbol{r})}) \leq \sum_{i=1}^{k} \operatorname{rank}\left(\overline{\mathcal{A}_{E_{i}}(\boldsymbol{r})}\right) \leq \sum_{i=1}^{k}\left(D\left|V\left(E_{i}\right)\right|-\right.$ $\left.D-\left|R\left(E_{i}\right)\right|\right)$. Since the proof is not short, the converse direction is left to the next subsection.

Corollary 4.13. Let $G=(V, E)$ be a graph with a bipartition $\mathcal{P}=\{B, R\}$ of $V$. If $d \geq 3$, then $\mathcal{M}(\mathcal{A}(\boldsymbol{r}))=\mathcal{M}_{f}(G, \mathcal{P})$ for almost all rod-configurations $\boldsymbol{r}$.

Proof. This directly follows from Theorem 4.12 and general results on polymatroids reviewed in section 2. Indeed, by Theorems 2.1 and 4.12 , the rank of $F \subseteq E$ in $\mathcal{M}(\mathcal{A}(\boldsymbol{r}))$ is written as

$$
\min \left\{\left|F_{0}\right|+\sum_{i=1}^{k}\left(D\left|V\left(F_{i}\right)\right|-D-\left|R\left(F_{i}\right)\right|\right)\right\}
$$

where the minimum is taken over all partitions $\left\{F_{0}, F_{1}, \ldots, F_{k}\right\}$ of $F$ such that $F_{1}, \ldots, F_{k} \neq \emptyset$. This is exactly the rank formula (2.3) of the matroid induced by $f$.

Combining Theorem 4.10 and Corollary 4.13, we conclude the proof of Theorem 4.9 .

Remark. Due to the absence of Lemma 4.6, the proof of Theorem 4.12 (given in the next subsection) could not be applied to the two-dimensional case. Although Theorem 4.12 can be proved even for the two-dimensional case with a slightly different manner, we will not go into detail as there are already many simpler proofs for this case $[21,39,35,41]$.

Theorem 4.9 is restated in terms of rigidity as follows.

Corollary 4.14. Let $G=(V, E)$ be a graph with a bipartition $\mathcal{P}=\{B, R\}$ of $V$. Then, there exists a bar-configuration $\boldsymbol{q}$ and a rod-configuration $\boldsymbol{r}$ such that the body-rod-bar framework $(G, \boldsymbol{q}, \boldsymbol{r})$ is minimally infinitesimally rigid (i.e., removing any bar results in a flexible framework) in $\mathbb{R}^{d}$ if and only if $G$ satisfies the following counting conditions:

- $|E|=D|B|+(D-1)|R|-D$;

- $|F| \leq D|B(F)|+(D-1)|R(F)|-D$ for any nonempty $F \subseteq E$.

Tay's combinatorial characterization of rod-bar frameworks is an easy consequence.

Corollary 4.15 (Tay $[32,31]$ ). Let $G=(V, E)$ be a graph. Then, there exists a bar-configuration $\boldsymbol{q}$ and a rod-configuration $\boldsymbol{r}$ such that the rod-bar framework $(G, \boldsymbol{q}, \boldsymbol{r})$ is minimally infinitesimally rigid in $\mathbb{R}^{d}$ if and only if $G$ satisfies the following counting conditions:

- $|E|=(D-1)|V|-D$;

- $|F| \leq(D-1)|V(F)|-D$ for any nonempty $F \subseteq E$.

Proof. The rod-bar framework $(G, \boldsymbol{q}, \boldsymbol{r})$ is a body-rod-bar framework with $R=V$ and $B=\emptyset$. In this case $D(|V(F)|-1)-|R(F)|=(D-1)|V(F)|-D$ for each $F \subseteq E$. Therefore, the statement follows from Corollary 4.14.

Copyright (c) by SIAM. Unauthorized reproduction of this article is prohibited. 
4.3. Proof of Theorem 4.12. We have already seen the $\leq$ direction of (4.6). The converse direction is proved by induction on the lexicographical ordering of the triples $(|V|,|R|,|E|)$. Since the base case is trivial, let us consider the general case. Since $A_{e}(r)=A_{e^{\prime}}(r)$ for any parallel $e$ and $e^{\prime}$, we may assume that $G$ is simple throughout the proof.

We split the proof into two cases depending on whether $B=\emptyset$.

4.3.1. Case of $B \neq \emptyset$. Let us first consider the easier case where there is a vertex $u \in B$. Let $N(u)=\left\{v_{1}, \ldots, v_{t}\right\}$ be the neighbors of $u$ in $G$. We remove $u$ and insert the edge set $K(N(u))$, that is, the edge set of the complete graph on $N(u)$. Let $H=\left(V-u, E \backslash \delta_{G}(u) \cup K(N(u))\right)$ be the resulting graph with the bipartition $\{B-u, R\}$ of $V-u$.

Let $\left\{E_{1}^{*}, \ldots, E_{k}^{*}\right\}$ be the $P$-connected component decomposition of $E(H)$ in $\mathcal{P} \mathcal{M}_{f}(H)$. By Lemma $4.5,\left\{E_{1}^{*}, \ldots, E_{k}^{*}\right\}$ is a minimizer of the right-hand side of (4.6) for $E(H)$. By induction, we have

$$
\operatorname{rank}\left(\overline{\left\{A_{e}(\boldsymbol{r}): e \in E(H)\right\}}\right)=\sum_{i=1}^{k} f\left(E_{i}^{*}\right)
$$

for almost all rod-configurations $\boldsymbol{r}: R \rightarrow G r(d-1, W)$.

If $N(u)=\{v\}$ for some $v \in V$, then $E=E(H)+u v$. It is easy to see $\mathcal{A}(\boldsymbol{r})=$ $\overline{\left\{A_{e}(\boldsymbol{r}): e \in E(H)\right\}} \oplus A_{u v}(\boldsymbol{r})$, and hence $\operatorname{rank}(\overline{\mathcal{A}(\boldsymbol{r})})=\operatorname{rank}\left(\overline{\left\{A_{e}(\boldsymbol{r}): e \in E(H)\right\}}\right)+$ $\operatorname{rank}\left(A_{u v}(\boldsymbol{r})\right)=\sum_{i=1}^{k} f\left(E_{i}^{*}\right)+f(\{u v\})$, implying the $\geq \operatorname{direction}$ of (4.6) since $\left\{E_{1}^{*}, \ldots\right.$, $\left.E_{k}^{*},\{u v\}\right\}$ is a partition of $E$.

Thus, let us assume $|N(u)| \geq 2$. Since $K(N(u))$ is a clique in $H$, it is straightforward to check that $K(N(u))$ is $P$-connected in $\mathcal{P} \mathcal{M}_{f}(H)$, and hence a $P$-connected component, say, $E_{k}^{*}$, contains $K(N(u))$ as a subset. This implies

$$
f\left(E_{k}^{*} \backslash K(N(u)) \cup \delta_{G}(u)\right)=f\left(E_{k}^{*}\right)+D .
$$

Observe that for any $v w \in K(N(u))$, we have

$$
A_{v w}(\boldsymbol{r}) \subseteq \overline{A_{v u}(\boldsymbol{r}) \cup A_{u w}(\boldsymbol{r})} .
$$

Indeed, any element of $A_{v w}(\boldsymbol{r})$ is written as

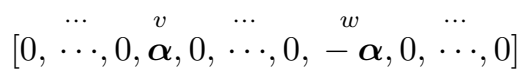

for some $\boldsymbol{\alpha} \in \mathbb{P}\left(\bigwedge^{2} W\right) \cap H_{\boldsymbol{r}}(v) \cap H_{\boldsymbol{r}}(w)$. This can be decomposed as

$$
\left[0, \cdots, 0, \stackrel{v}{\boldsymbol{\alpha}}, 0, \cdots, 0,{ }^{u} \boldsymbol{\alpha}, 0, \cdots, 0\right]+\left[0, \cdots, 0, \stackrel{u}{\boldsymbol{\alpha}}, 0, \cdots, 0,{ }^{w} \boldsymbol{\alpha}, 0, \cdots, 0\right],
$$

where these two terms are contained in $A_{v u}(\boldsymbol{r})$ and $A_{u w}(\boldsymbol{r})$, respectively, because $H_{\boldsymbol{r}}(u)=\mathbb{P}\left(\bigwedge^{2} W\right)$ by $u \in B$.

Equation (4.9) implies $\overline{\left\{A_{e}(\boldsymbol{r}): e \in E(H)\right\}} \subseteq \overline{\mathcal{A}(\boldsymbol{r})}$. Moreover, we can always take independent $D$ points $p_{1}, \ldots, p_{D}$ from $\left\{A_{e}(\boldsymbol{r}): e \in \delta(u)\right\}$ since $u \in B$ and $|N(u)| \geq 2$.

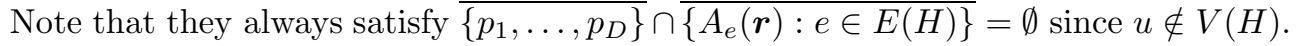
We thus obtain

$$
\operatorname{rank}(\overline{\mathcal{A}(\boldsymbol{r})}) \geq \operatorname{rank}\left(\overline{\left\{A_{e}(\boldsymbol{r}): e \in E(H)\right\}}\right)+D .
$$

Combining (4.8), (4.7), and (4.10), we obtain $\operatorname{rank}(\overline{\mathcal{A}(\boldsymbol{r})}) \geq \sum_{i=1}^{k-1} f\left(E_{i}^{*}\right)+f\left(E_{k}^{*} \backslash\right.$ $K(N(u)) \cup \delta(u))$, implying the $\geq$ direction of (4.6) since $\left\{E_{1}^{*}, \ldots, E_{k-1}^{*}, E_{k}^{*} \backslash K(N(u)) \cup\right.$ $\delta(u)\}$ is a partition of $E$. This completes the proof for case $B \neq \emptyset$. 
4.3.2. Case of $\boldsymbol{B}=\emptyset$. For any $u \in V$, let $\mathcal{P}_{u}=\{B+u, R-u\}$. Note that, by induction, the linear polymatroid $\mathcal{P} \mathcal{M}\left(\mathcal{A}\left(\boldsymbol{r}^{\prime}\right)\right)$ is equal to $\mathcal{P} \mathcal{M}_{f}\left(G, \mathcal{P}_{u}\right)$ for almost all rod-configurations $\boldsymbol{r}^{\prime}$ on $R-u$. Our proof is based on this inductive relation. Intuitively speaking, we will replace a body associated with $u$ by a rod $\boldsymbol{r}(u)$. This operation corresponds with restricting $\mathbb{P}\left(V_{u}\right)=\mathbb{P}\left(\bigwedge^{2} W\right)$ to a hyperplane $H_{\boldsymbol{r}}(u)$ of $\mathbb{P}\left(\bigwedge^{2} W\right)$, which is the dual of the point $\boldsymbol{r}(u)$. This operation is equivalent to the restriction of $\mathcal{A}\left(\boldsymbol{r}^{\prime}\right)$ to a special hyperplane $H$ in $\mathbb{P}\left(V_{V}\right)$ such that $H \cap \mathbb{P}\left(V_{u}\right)=H_{\boldsymbol{r}}(u)$ and $\mathbb{P}\left(V_{v}\right) \subset H$ for all $v \in V-u$. This hyperplane $H$ is not generic within $\mathbb{P}\left(V_{V}\right)$ (and hence this operation is not Dilworth truncation), but we may take $H$ so that $H \cap \mathbb{P}\left(V_{u}\right)$ is generic within $\mathbb{P}\left(V_{u}\right)$. We will show that the naturally extended rank formula of Dilworth truncation holds for this operation for some $u \in V$.

The proof consists of a sequence of lemmas. We first define a generic hyperplane within $\mathbb{P}\left(V_{u}\right)$ for a vertex $u \in V$ and show the existence of generic hyperplanes in Lemma 4.16. We then discuss about an extension of a rod-configuration $\boldsymbol{r}^{\prime}: R-u \rightarrow$ $G r(d-1, W)$ to $\boldsymbol{r}: R \rightarrow G r(d-1, W)$, where $\boldsymbol{r}$ is said to be an extension of $\boldsymbol{r}^{\prime}$ if $\boldsymbol{r}(v)=\boldsymbol{r}^{\prime}(v)$ for all $v \in V-u$. We shall define a generic extension of a rodconfiguration based on a generic hyperplane in $\mathbb{P}\left(V_{u}\right)$. Then in Lemma 4.18 we shall show an existence of a vertex $u \in V$ having special properties and finally perform a variant of Dilworth truncation at $u$ in Lemma 4.19.

For a flat $A$ of $\mathbb{P}\left(V_{V}\right)$ and a vertex $u \in V$, $\operatorname{proj}_{u}(A)$ denotes the orthogonal projection ${ }^{2}$ of $A$ onto $\mathbb{P}\left(V_{u}\right)$. A hyperplane $H_{u}$ of $\mathbb{P}\left(V_{u}\right)$ is called generic relative to a finite set $\mathcal{A}$ of flats in $\mathbb{P}\left(V_{V}\right)$ if it satisfies the following property; for every $\mathcal{A}_{1}, \mathcal{A}_{2} \subseteq \mathcal{A}$ with $\operatorname{proj}_{u}\left(\overline{\mathcal{A}_{1}} \cap \overline{\mathcal{A}_{2}}\right) \neq \emptyset$ (where we allow $\mathcal{A}_{1}=\mathcal{A}_{2}$ ),

$$
\operatorname{rank}\left(\operatorname{proj}_{u}\left(\overline{\mathcal{A}_{1}} \cap \overline{\mathcal{A}_{2}}\right) \cap H_{u}\right)=\operatorname{rank}\left(\operatorname{proj}_{u}\left(\overline{\mathcal{A}_{1}} \cap \overline{\mathcal{A}_{2}}\right)\right)-1 .
$$

The next lemma shows the existence of generic hyperplanes.

Lemma 4.16. Let $u \in V$ and $\mathcal{A}$ be a finite set of flats in $\mathbb{P}\left(V_{V}\right)$. Suppose $G r(d-1, W) \subseteq \mathbb{P}\left(V_{u}\right)$ (by identifying $V_{u}$ with $\bigwedge^{d-1} W$ ). Then, for almost all points $\left[\boldsymbol{r}_{u}\right] \in G r(d-1, W)$, the hyperplane $H_{u}$ of $\mathbb{P}\left(V_{u}\right)$ dual to $\left[\boldsymbol{r}_{u}\right]$ is generic relative to $\mathcal{A}$.

Proof. Take any $\mathcal{A}_{1}, \mathcal{A}_{2} \subseteq \mathcal{A}$ with $\operatorname{proj}_{u}\left(\overline{\mathcal{A}_{1}} \cap \overline{\mathcal{A}_{2}}\right) \neq \emptyset$, and let us denote $A=$ $\overline{\mathcal{A}_{1}} \cap \overline{\mathcal{A}_{2}}$ for simplicity. It is clear that $\operatorname{rank}\left(\operatorname{proj}_{u}(A) \cap H_{u}\right) \geq \operatorname{rank}\left(\operatorname{proj}_{u}(A)\right)-1$ for any

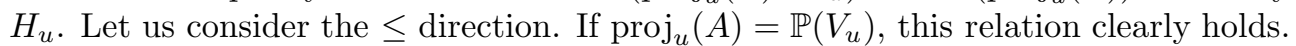
Otherwise $\operatorname{proj}_{u}(A)$ is a linear subspace of $\mathbb{P}\left(V_{u}\right)$, and hence $\operatorname{rank}\left(\operatorname{proj}_{u}(A) \cap H_{u}\right) \leq$ $\operatorname{rank}\left(\operatorname{proj}_{u}(A)\right)-1$ holds if we take $\left[\boldsymbol{r}_{u}\right] \in \operatorname{Gr}(d-1, W)$ so that $\left[\boldsymbol{r}_{u}\right]$ is not contained in the dual of $\operatorname{proj}_{u}(A)$ in $\mathbb{P}\left(V_{u}\right)$. Since the intersection of the dual of $\operatorname{proj}_{u}(A)$ with $\operatorname{Gr}(d-1, W)$ is a lower-dimensional subvariety of $G r(d-1, W)$, almost all $\left[\boldsymbol{r}_{u}\right]$ satisfy this property.

Since there are a finite number of possible $A=\overline{\mathcal{A}_{1}} \cap \overline{\mathcal{A}_{2}}$, almost all hyperplanes $H_{u}$ of $\mathbb{P}\left(V_{u}\right)$ are indeed generic.

We now define a generic extension of a rod-configuration $\boldsymbol{r}^{\prime}: R-u \rightarrow G r(d-1, W)$ as follows: a rod-configuration $\boldsymbol{r}: R \rightarrow G r(d-1, W)$ is a generic extension of $\boldsymbol{r}^{\prime}$ if $\boldsymbol{r}(v)=\boldsymbol{r}^{\prime}(v)$ for $v \in V-u$ and $\boldsymbol{r}(u)$ satisfies the property that the dual hyperplane of $\boldsymbol{r}(u)$ in $\mathbb{P}\left(V_{u}\right)$ is generic relative to $\mathcal{A}\left(\boldsymbol{r}^{\prime}\right)$. By Lemma 4.16, almost all extensions are generic.

Once we pick out a generic extension $\boldsymbol{r}$ of $\boldsymbol{r}^{\prime}$, the unique hyperplane $H$ of $\mathbb{P}\left(V_{V}\right)$ is determined in such a way that $H \cap \mathbb{P}\left(V_{u}\right)$ is the dual hyperplane of $\boldsymbol{r}(u)$ in $\mathbb{P}\left(V_{u}\right)$

\footnotetext{
${ }^{2}$ More precisely, let $W^{\prime}$ be the linear subspace of $V_{V}$ satisfying $A=\mathbb{P}\left(W^{\prime}\right)$, and let $\operatorname{proj}_{u}\left(W^{\prime}\right)$ be the orthogonal projection of $W^{\prime}$ onto $V_{u}$. We define $\operatorname{proj}_{u}(A)$ by $\mathbb{P}\left(\operatorname{proj}_{u}\left(W^{\prime}\right)\right)$.
} 
and $\mathbb{P}\left(V_{v}\right) \subset H$ for all $v \in V-u$. Such a unique hyperplane $H$ is called the hyperplane associated with the generic extension.

It is important to observe

$$
A_{e}(\boldsymbol{r})=A_{e}\left(\boldsymbol{r}^{\prime}\right) \cap H \quad \text { for every } e \in E .
$$

Also, if we define $\chi_{u}$ by

$$
\chi_{u}(A)= \begin{cases}1 & \text { if } \operatorname{proj}_{u}(A) \neq \emptyset \\ 0 & \text { otherwise }\end{cases}
$$

for a flat $A \subset \mathbb{P}\left(V_{V}\right)$, then we have the following from the genericity (4.11): for every $\mathcal{A}_{1}, \mathcal{A}_{2} \subseteq \mathcal{A}\left(\boldsymbol{r}^{\prime}\right)$

$$
\operatorname{rank}\left(\left(\overline{\mathcal{A}_{1}} \cap \overline{\mathcal{A}_{2}}\right) \cap H\right)=\operatorname{rank}\left(\overline{\mathcal{A}_{1}} \cap \overline{\mathcal{A}_{2}}\right)-\chi_{u}\left(\overline{\mathcal{A}_{1}} \cap \overline{\mathcal{A}_{2}}\right) .
$$

Note that, setting $\mathcal{A}_{1}=\mathcal{A}_{2},(4.13)$ implies, for every $\mathcal{A}_{1} \subseteq \mathcal{A}\left(\boldsymbol{r}^{\prime}\right)$

$$
\operatorname{rank}\left(\overline{\mathcal{A}_{1}} \cap H\right)=\operatorname{rank}\left(\overline{\mathcal{A}_{1}}\right)-\chi_{u}\left(\overline{\mathcal{A}_{1}}\right) .
$$

In particular, for any $A_{e}\left(\boldsymbol{r}^{\prime}\right) \in \mathcal{A}\left(\boldsymbol{r}^{\prime}\right)$,

$$
\operatorname{rank}\left(A_{e}\left(\boldsymbol{r}^{\prime}\right) \cap H\right)=\operatorname{rank}\left(A_{e}\left(\boldsymbol{r}^{\prime}\right)\right)-\chi_{u}\left(A_{e}\left(\boldsymbol{r}^{\prime}\right)\right) .
$$

By (4.12), our goal is now to extend Theorem 2.2 to the case of our special hyperplane $H$. Such an extension will be given in Lemma 4.19 by performing a truncation at a vertex $u$ shown in the following lemma (Lemma 4.18). Before that, we need an easy observation.

Lemma 4.17. Let $C$ be a $P$-connected set in $\mathcal{P} \mathcal{M}(G, \mathcal{P})$ with $C \neq E$. Then, for almost all rod-configurations $\boldsymbol{r}$ on $R, \mathcal{A}_{C}(\boldsymbol{r})$ is connected.

Proof. Let us consider the restriction to $C$, i.e., consider $G^{\prime}=(V(C), C), \mathcal{P}^{\prime}=$ $\{B \cap V(C), R \cap V(C)\}$. Note that $|V(C)| \leq|V|,|R \cap V(C)| \leq|R|$, and $|C|<|E|$ by $C \subseteq E-e$. Hence, by induction, the linear polymatroid $\mathcal{P} \mathcal{M}\left(\mathcal{A}_{C}(\boldsymbol{r})\right)$ is equal to $\mathcal{P} \mathcal{M}_{f}\left(G^{\prime}, \mathcal{P}^{\prime}\right)$ for almost all rod-configurations $\boldsymbol{r}$ on $R$. Since $C$ is a $P$-connected in $\mathcal{P} \mathcal{M}_{f}\left(G^{\prime}, \mathcal{P}^{\prime}\right), \mathcal{A}_{C}(\boldsymbol{r})$ is connected.

LEMma 4.18. There exists a vertex $u$ satisfying one of the following two properties: For almost all rod-configurations $\boldsymbol{r}^{\prime}$ on $R-u$ and almost all extension $\boldsymbol{r}$,

(A) $G$ has an edge subset $C$ with $\delta_{G}(u) \subset C \subsetneq E$ such that $\mathcal{A}_{C}(\boldsymbol{r})$ is connected; or

(B) $G$ has disjoint edge subsets $C$ and $C^{\prime}$ with $\delta_{G}(u) \subset C \cup C^{\prime}$ such that both $\mathcal{A}_{C}(\boldsymbol{r})$ and $\mathcal{A}_{C^{\prime}}(\boldsymbol{r})$ are connected. Furthermore, if $\mathcal{A}\left(\boldsymbol{r}^{\prime}\right)$ is connected, then $\operatorname{proj}_{u}\left(\overline{\mathcal{A}_{C}\left(\boldsymbol{r}^{\prime}\right)} \cap \overline{\mathcal{A}_{E \backslash C}\left(\boldsymbol{r}^{\prime}\right)}\right) \neq \emptyset$.

Proof. Take any edge $e \in E$, and consider $G$-e. By Lemma 4.6, G-e has (i) three vertices each of which is spanned by two $P$-connected components of $\mathcal{P} \mathcal{M}(G-e, \mathcal{P})$, or (ii) a vertex spanned by exactly one $P$-connected component of $\mathcal{P} \mathcal{M}(G-e, \mathcal{P})$. Since any $P$-connected set of $\mathcal{P} \mathcal{M}(G-e, \mathcal{P})$ is also $P$-connected in $\mathcal{P} \mathcal{M}(G, \mathcal{P})$, these $P$-connected components are $P$-connected in $\mathcal{P M}(G, \mathcal{P})$.

We define $C, C^{\prime} \subseteq E-e$ as follows: If (i) occurs, then take a vertex $u$ that is not an endpoint of $e$ and is spanned by two $P$-connected components in $\mathcal{P} \mathcal{M}(G-e, \mathcal{P})$. Let $C$ and $C^{\prime}$ be such components. If (ii) occurs, then we have a vertex $u$ spanned by exactly one $P$-connected component in $\mathcal{P M}(G-e, \mathcal{P})$. Let $C$ be that component. Furthermore, if $u$ is an endpoint of $e$, let $C^{\prime}=\{e\}$. 
Consequently, one of the followings holds: (i') $C$ is $P$-connected set with $\delta_{G}(u) \subset$ $C \subsetneq E$ or (ii') $C$ and $C^{\prime}$ are disjoint $P$-connected sets (that may be trivial) with $\delta_{G}(u) \subset C \cup C^{\prime}$. Note that both $C$ and $C^{\prime}$ are proper subsets of $E$, and thus Lemma 4.17 implies that $\mathcal{A}_{C}(\boldsymbol{r})$ and $\mathcal{A}_{C^{\prime}}(\boldsymbol{r})$ are connected for almost all rodconfigurations $\boldsymbol{r}$.

The remaining thing is to prove the last property of $(\mathrm{B})$ when (ii') occurs. Recall $\mathcal{P}_{u}=\{B+u, R-u\}$, and the linear polymatroid $\mathcal{P} \mathcal{M}\left(\left\{A_{e}\left(r^{\prime}\right): e \in K(V)\right\}\right)$ is equal to $\mathcal{P M}_{f}\left(K(V), \mathcal{P}_{u}\right)$ by induction on the lexicographical order of $(|V|,|R|,|E|)$. Since $\mathcal{A}\left(\boldsymbol{r}^{\prime}\right)$ is connected, $E$ is $P$-connected in $\mathcal{P} \mathcal{M}_{f}\left(G, \mathcal{P}_{u}\right)$. Thus, applying Lemma 4.8, we may assume that there is a vertex $v \in V-u$ with $u v \in \operatorname{cl}(C) \cap \operatorname{cl}(E \backslash C)$ for the closure operator of $\mathcal{P} \mathcal{M}_{f}\left(G, \mathcal{P}_{u}\right)$. This implies $\left.A_{u v}\left(\boldsymbol{r}^{\prime}\right) \subset \overline{\mathcal{A}_{C}\left(\boldsymbol{r}^{\prime}\right)} \cap \overline{\mathcal{A}_{E \backslash C}\left(\boldsymbol{r}^{\prime}\right)}\right)$, and thus $\operatorname{proj}_{u}\left(\overline{\mathcal{A}_{C}\left(\boldsymbol{r}^{\prime}\right)} \cap \overline{\mathcal{A}_{E \backslash C}\left(\boldsymbol{r}^{\prime}\right)}\right) \neq \emptyset$.

We are now ready to extend Theorem 2.2 to our nongeneric hyperplane. Recall that for a family $\mathcal{A}$ of flats and a hyperplane $H$, we abbreviate $\{A \cap H: A \in \mathcal{A}\}$ as $\mathcal{A} \cap H$. Note that $(\overline{\mathcal{A}}) \cap H$ implies $\overline{\{A: A \in \mathcal{A}\}} \cap H$, which may not be equal to $\overline{\mathcal{A} \cap H}=\overline{\{A \cap H: A \in \mathcal{A}\}}$.

LEMma 4.19. Let $u$ be a vertex shown in Lemma 4.18 and $\boldsymbol{r}^{\prime}$ be a generic rodconfiguration on $R-u$. Then, for the hyperplane $H$ of $\mathbb{P}\left(V_{V}\right)$ associated with a generic extension of $\boldsymbol{r}^{\prime}$,

$$
\operatorname{rank}\left(\overline{\mathcal{A}\left(\boldsymbol{r}^{\prime}\right) \cap H}\right)=\min \left\{\sum_{i=1}^{k}\left(\operatorname{rank}\left(\overline{\mathcal{A}_{E_{i}}\left(\boldsymbol{r}^{\prime}\right)}\right)-\chi_{u}\left(\overline{\mathcal{A}_{E_{i}}\left(\boldsymbol{r}^{\prime}\right)}\right)\right)\right\},
$$

where the minimum is taken over all partitions $\left\{E_{1}, \ldots, E_{k}\right\}$ of $E$ into nonempty subsets.

Proof. For simplicity, we abbreviate $A_{e}\left(\boldsymbol{r}^{\prime}\right)$ as $A_{e}^{\prime}$ and $\mathcal{A}\left(\boldsymbol{r}^{\prime}\right)$ as $\mathcal{A}^{\prime}$, respectively. Consider the connected component decomposition of $\mathcal{A}^{\prime} \cap H$ (that is, the $P$-connected component decomposition of the linear polymatroid $\left.\mathcal{P} \mathcal{M}\left(\mathcal{A}^{\prime} \cap H\right)\right)$. To see the equality of (4.16), we show (4.16) for each connected component of $\mathcal{A}^{\prime} \cap H$ Thus, by induction, we may assume $\mathcal{A}^{\prime} \cap H$ is connected and it is sufficient to show

$$
\operatorname{rank}\left(\overline{\mathcal{A}^{\prime} \cap H}\right)=\operatorname{rank}\left(\overline{\mathcal{A}^{\prime}}\right)-1 .
$$

From the choice of $u,(\mathrm{~A})$ or (B) of Lemma 4.18 holds. Let $C$ and $C^{\prime}$ be subsets of $E$ satisfying properties of Lemma 4.18, where $C^{\prime}=\emptyset$ if (A) holds (otherwise we may assume $\left.C^{\prime} \neq \emptyset\right)$. Namely, if $C^{\prime}=\emptyset, \mathcal{A}_{C}^{\prime} \cap H$ is connected with $\delta_{G}(u) \subseteq C$. (Note that in the current situation, $\mathcal{A}_{C}^{\prime} \cap H$ corresponds to $\mathcal{A}_{C}(\boldsymbol{r})$ of the statement of Lemma 4.18).) If $C^{\prime} \neq \emptyset, \mathcal{A}_{C}^{\prime} \cap H$ and $\mathcal{A}_{C^{\prime}}^{\prime} \cap H$ are connected with $\delta_{G}(u) \subseteq C \cup C^{\prime}$. We may further assume $\delta_{G}(u) \cap C \neq \emptyset$ and $\delta_{G}(u) \cap C^{\prime} \neq \emptyset$, since otherwise we have the former case.

We now calculate the rank of $\overline{\mathcal{A}_{C}^{\prime} \cap H}, \overline{\mathcal{A}_{C^{\prime}}^{\prime} \cap H}$, and $\overline{\mathcal{A}_{E \backslash C}^{\prime} \cap H}$. The connectivity of $\mathcal{A}_{C}^{\prime} \cap H$ and $\delta_{G}(u) \cap C$ implies

$$
\operatorname{rank}\left(\overline{\mathcal{A}_{C}^{\prime} \cap H}\right)=\operatorname{rank}\left(\overline{\mathcal{A}_{C}^{\prime}}\right)-1
$$

by induction. Similarly, if $C^{\prime} \neq \emptyset$, the connectivity of $\mathcal{A}_{C^{\prime}}^{\prime} \cap H$ and $\delta_{G}(u) \cap C^{\prime}$ implies

$$
\operatorname{rank}\left(\overline{\mathcal{A}_{C^{\prime}}^{\prime} \cap H}\right)=\operatorname{rank}\left(\overline{\mathcal{A}_{C^{\prime}}^{\prime}}\right)-1 .
$$

Also, since all flats of $\mathcal{A}_{E \backslash\left(C \cup C^{\prime}\right)}^{\prime}$ are contained in $H$ by $\delta_{G}(u) \subset C \cup C^{\prime}$, we have

$$
\mathcal{A}_{E \backslash\left(C \cup C^{\prime}\right)}^{\prime} \cap H=\mathcal{A}_{E \backslash\left(C \cup C^{\prime}\right)}^{\prime} .
$$


Suppose $C^{\prime} \neq \emptyset$, and let us take an edge $e \in \delta_{G}(u) \cap C^{\prime}$ and a point $x \in A_{e}^{\prime} \backslash$ $H$. (Note that by $(4.15), A_{e}^{\prime} \backslash H \neq \emptyset$.) Then, clearly $\operatorname{rank}\left(\overline{\overline{\left(\mathcal{A}_{C^{\prime}}^{\prime} \cap H\right)} \cup\{x\}}\right)=$ $\operatorname{rank}\left(\overline{\mathcal{A}_{C^{\prime}}^{\prime} \cap H}\right)+1$. Combined with (4.19), we have

$$
\overline{\mathcal{A}_{C^{\prime}}^{\prime}}=\overline{\overline{\left(\mathcal{A}_{C^{\prime}}^{\prime} \cap H\right)} \cup\{x\}} \text {. }
$$

By (4.20) and (4.21),

$$
\begin{aligned}
\overline{\mathcal{A}_{E \backslash C}^{\prime}} \cap H & =\overline{\mathcal{A}_{E \backslash\left(C \cup C^{\prime}\right)}^{\prime} \cup \mathcal{A}_{C^{\prime}}^{\prime}} \cap H \\
& =\overline{\left(\overline{\mathcal{A}_{E \backslash\left(C \cup C^{\prime}\right.}^{\prime} \cap H}\right) \cup\left(\overline{\mathcal{A}_{C^{\prime}}^{\prime} \cap H}\right) \cup\{x\}} \cap H \\
& =\overline{\left(\overline{\mathcal{A}_{E \backslash\left(C \cup C^{\prime}\right.}^{\prime} \cap H}\right) \cup\left(\overline{\mathcal{A}_{C^{\prime}}^{\prime} \cap H}\right)}=\overline{\mathcal{A}_{E \backslash C}^{\prime} \cap H} .
\end{aligned}
$$

Thus, applying (4.14), we obtain

$$
\operatorname{rank}\left(\overline{\mathcal{A}_{E \backslash C}^{\prime} \cap H}\right)=\operatorname{rank}\left(\overline{\mathcal{A}_{E \backslash C}^{\prime}} \cap H\right)=\operatorname{rank}\left(\overline{\mathcal{A}_{E \backslash C}^{\prime}}\right)-1
$$

if $C^{\prime} \neq \emptyset$. In total, combining (4.20) and (4.23),

$$
\operatorname{rank}\left(\overline{\mathcal{A}_{E \backslash C}^{\prime} \cap H}\right)= \begin{cases}\operatorname{rank}\left(\overline{\mathcal{A}_{E \backslash C}^{\prime}}\right)-1 & \left(\text { if } C^{\prime} \neq \emptyset\right), \\ \operatorname{rank}\left(\overline{\mathcal{A}_{E \backslash C}^{\prime}}\right) & \text { (if } \left.C^{\prime}=\emptyset\right) .\end{cases}
$$

We then compute the rank of $\left(\overline{\mathcal{A}_{C}^{\prime} \cap H}\right) \cap\left(\overline{\mathcal{A}_{E \backslash C}^{\prime} \cap H}\right)$. Since $\operatorname{rank}\left(\left(\overline{\mathcal{A}_{C}^{\prime}}\right) \cap H\right)=$ $\operatorname{rank}\left(\overline{\mathcal{A}_{C}^{\prime}}\right)-1$ by (4.14), comparing this relation with (4.18), we have

$$
\overline{\mathcal{A}_{C}^{\prime}} \cap H=\overline{\mathcal{A}_{C}^{\prime} \cap H} \text {. }
$$

By (4.22) and (4.25), we obtain

$$
\left(\overline{\mathcal{A}_{C}^{\prime} \cap H}\right) \cap\left(\overline{\mathcal{A}_{E \backslash C}^{\prime} \cap H}\right)=\left(\overline{\mathcal{A}_{C}^{\prime}}\right) \cap\left(\overline{\mathcal{A}_{E \backslash C}^{\prime}}\right) \cap H .
$$

Therefore, applying (4.26) and then (4.13), we obtain

$$
\begin{aligned}
\operatorname{rank}\left(\left(\overline{\mathcal{A}_{C}^{\prime} \cap H}\right) \cap\left(\overline{\mathcal{A}_{E \backslash C}^{\prime} \cap H}\right)\right) & =\operatorname{rank}\left(\left(\overline{\mathcal{A}_{C}^{\prime}}\right) \cap\left(\overline{\mathcal{A}_{E \backslash C}^{\prime}}\right) \cap H\right) \\
& =\operatorname{rank}\left(\overline{\mathcal{A}_{C}^{\prime}} \cap \overline{\mathcal{A}_{E \backslash C}^{\prime}}\right)-\chi_{u}\left(\overline{\mathcal{A}_{C}^{\prime}} \cap \overline{\mathcal{A}_{E \backslash C}^{\prime}}\right) .
\end{aligned}
$$

We show that $\chi_{u}\left(\overline{\mathcal{A}_{C}^{\prime}} \cap \overline{\mathcal{A}_{E \backslash C}^{\prime}}\right)$ takes distinct values depending on whether $C^{\prime}=\emptyset$. If $C^{\prime}=\emptyset$, then no edge in $E \backslash C$ is incident to $u$ by $\delta_{G}(u) \subseteq C$, and $\operatorname{proj}_{u}\left(\overline{\mathcal{A}_{E \backslash C}^{\prime}}\right)=\emptyset$. Thus, $\chi_{u}\left(\overline{\mathcal{A}_{C}^{\prime}} \cap \overline{\mathcal{A}_{E \backslash C}^{\prime}}\right)=0$. On the other hand, if $C^{\prime} \neq \emptyset$, then property (B) of Lemma 4.18 implies $\operatorname{proj}_{u}\left(\overline{\mathcal{A}_{C}^{\prime}} \cap \overline{\mathcal{A}_{E \backslash C}}\right) \neq \emptyset$ since $\mathcal{A}^{\prime}$ is connected from the connectivity of $\mathcal{A}^{\prime} \cap H$. Therefore, $\chi_{u}\left(\overline{\mathcal{A}_{C}^{\prime}} \cap \overline{\mathcal{A}_{E \backslash C}^{\prime}}\right)=1$ if $C^{\prime} \neq \emptyset$. In total, (4.27) can be rewritten by

$$
\operatorname{rank}\left(\left(\overline{\mathcal{A}_{C}^{\prime} \cap H}\right) \cap\left(\overline{\mathcal{A}_{E \backslash C}^{\prime} \cap H}\right)\right)= \begin{cases}\operatorname{rank}\left(\overline{\mathcal{A}_{C}^{\prime}} \cap \overline{\mathcal{A}_{E \backslash C}^{\prime}}\right)-1 & \left(\text { if } C^{\prime} \neq \emptyset\right), \\ \operatorname{rank}\left(\overline{\mathcal{A}_{C}^{\prime}} \cap \overline{\mathcal{A}_{E \backslash C}^{\prime}}\right) & \left(\text { if } C^{\prime}=\emptyset\right) .\end{cases}
$$

By (4.18), (4.24), (4.28), and the modularity of $\operatorname{rank}(\cdot)$,

$$
\begin{aligned}
\operatorname{rank}\left(\overline{\mathcal{A}^{\prime} \cap H}\right) & =\operatorname{rank}\left(\overline{\left(\overline{\mathcal{A}_{C}^{\prime} \cap H}\right) \cup\left(\overline{\mathcal{A}_{E \backslash C}^{\prime} \cap H}\right)}\right) \\
& \left.=\operatorname{rank}\left(\overline{\mathcal{A}_{C}^{\prime} \cap H}\right)+\operatorname{rank}\left(\overline{\mathcal{A}_{E \backslash C}^{\prime} \cap H}\right)-\operatorname{rank}\left(\overline{\left(\mathcal{A}_{C}^{\prime} \cap H\right.}\right) \cap\left(\overline{\mathcal{A}_{E \backslash C}^{\prime} \cap H}\right)\right) \\
& =\operatorname{rank}\left(\overline{\mathcal{A}_{C}^{\prime}}\right)+\operatorname{rank}\left(\overline{\mathcal{A}_{E \backslash C}^{\prime}}\right)-\operatorname{rank}\left(\overline{\mathcal{A}_{C}^{\prime}} \cap \overline{\mathcal{A}_{E \backslash C}^{\prime}}\right)-1 \\
& =\operatorname{rank}\left(\overline{\mathcal{A}^{\prime}}\right)-1,
\end{aligned}
$$

implying (4.17). This completes the proof of the lemma. 
For $F \subseteq E$, let

$$
\chi_{u}(F)= \begin{cases}1 & \text { if } u \in V(F) \\ 0 & \text { otherwise }\end{cases}
$$

where $u$ is a vertex shown in Lemma 4.18. Then, Lemma 4.19 implies, for almost all bar-configurations $\boldsymbol{r}^{\prime}$ on $R-u$ and its generic extension $\boldsymbol{r}$,

$$
\operatorname{rank}(\overline{\mathcal{A}(\boldsymbol{r})})=\min \left\{\sum_{i}\left(\operatorname{rank}\left(\overline{\mathcal{A}_{E_{i}}\left(\boldsymbol{r}^{\prime}\right)}\right)-\chi_{u}\left(E_{i}\right)\right): \text { a partition }\left\{E_{1}, \ldots, E_{k}\right\} \text { of } E\right\} \text {. }
$$

Let $R^{\prime}=R-u$. The induction hypothesis on $|R|$ implies

$$
\begin{aligned}
& \operatorname{rank}\left(\overline{\mathcal{A}_{E_{i}}\left(\boldsymbol{r}^{\prime}\right)}\right) \\
& =\min \left\{\sum_{j}\left(D\left|V\left(E_{i, j}\right)\right|-D-\left|R^{\prime}\left(E_{i, j}\right)\right|\right): \text { a partition }\left\{E_{i, 1}, \ldots, E_{i, k^{\prime}}\right\} \text { of } E_{i}\right\}
\end{aligned}
$$

for each $E_{i} \subseteq E$. Since $\chi_{u}\left(E_{i}\right) \leq \sum_{j} \chi_{u}\left(E_{i, j}\right)$ for any $E_{i} \subseteq E$ and any partition $\left\{E_{i, 1}, \ldots, E_{i, k^{\prime}}\right\}$ of $E_{i},(4.29)$ and (4.30) imply

$$
\begin{aligned}
& \operatorname{rank}(\overline{\mathcal{A}(\boldsymbol{r})}) \\
& \quad \geq \min \left\{\sum_{i}\left(D\left|V\left(E_{i}\right)\right|-D-\left|R^{\prime}\left(E_{i}\right)\right|-\chi_{u}\left(E_{i}\right)\right) \text { : a partition }\left\{E_{1}, \ldots, E_{k}\right\} \text { of } E\right\} .
\end{aligned}
$$

Note that for any $F \subseteq E$, we have $|R(F)|=\left|R^{\prime}(F)\right|+\chi_{u}(F)$. Thus, (4.31) implies the $\geq$ direction of (4.6) for case $B=\emptyset$. This completes the proof of Theorem 4.12 .

5. Identified body-hinge frameworks. An identified body-hinge framework (simply called a body-hinge framework) is a structure consisting of rigid bodies connected by hinges (that is, $(d-2)$-dimensional flats). A hinge is allowed to connect any number of bodies. A body-hinge framework is formally defined as a pair $(G, \boldsymbol{h})$, where

- $G=(B, H ; E)$ is a bipartite graph with vertex classes $B$ and $H$, representing bodies and hinges, respectively;

- $\boldsymbol{h}: H \rightarrow G r(d-1, W)$ is a hinge-configuration.

Note that each $v_{1} \in B$ and $v_{2} \in H$ correspond to a body and a hinge, respectively, and $e \in E$ indicates their incidence.

A motion of $(G, \boldsymbol{h})$ is defined as a mapping $\boldsymbol{m}: B \rightarrow \bigwedge^{d-1} W$ such that $\boldsymbol{m}(u)-$ $\boldsymbol{m}(v)$ is contained in $\boldsymbol{h}(w)$ for any neighbors $u, v \in B$ of $w \in H$. A motion $\boldsymbol{m}$ is called trivial if $\boldsymbol{m}(v)$ 's are equal for all $v \in B .(G, \boldsymbol{h})$ is said to be infinitesimally rigid if every motion is trivial.

For a bipartite graph $G=(B, H ; E)$, the graph obtained from $G$ by duplicating each edge by $(D-1)$ parallel copies is denoted by $(D-1) \circ G$, and $(D-1) \circ E$ denotes the edge set of $(D-1) \circ G$. Tay showed a combinatorial characterization of identified body-hinge frameworks by converting to rod-bar frameworks. Below, we give a more natural proof. 

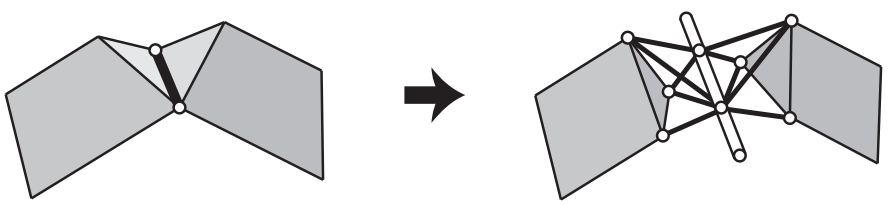

FIG. 5.1. Conversion of the body-hinge model to the body-rod-bar model.

Corollary 5.1 (Tay [31]). Let $G=(B, H ; E)$ be a bipartite graph. Then, there exists a hinge-configuration $\boldsymbol{h}$ such that $(G, \boldsymbol{h})$ is infinitesimally rigid if and only if $(D-1) \circ G$ contains an edge subset $I \subseteq(D-1) \circ E$ satisfying the following counting conditions:

- $|I|=D|B|+(D-1)|H|-D$;

- $|F| \leq D|B(F)|+(D-1)|H(F)|-D$ for each nonempty $F \subseteq I$.

Proof. Let $(G, \boldsymbol{h})$ be an identified body-hinge framework. For an edge $e=u v \in E$ with $u \in H$ and $v \in B$, we can regard $\boldsymbol{h}(u)$ as a rod (generically) linked by $(D-1)$ bars with the body associated with $v$ (see Figure 5.1). Hence, the identified body-hinge framework $(G, \boldsymbol{h})$ is equal to the body-rod-bar framework $\left(G^{\prime}, \boldsymbol{q}, \boldsymbol{r}\right)$, where $G^{\prime}$ is the graph with $V\left(G^{\prime}\right)=B \cup H$ and $E\left(G^{\prime}\right)=(D-1) \circ E, \boldsymbol{r}=\boldsymbol{h}$, and $\boldsymbol{q}$ is a generic barconfiguration. Since $D(|B(F) \cup H(F)|-1)-|H(F)|=D|B(F)|+(D-1)|H(F)|-D$ for any $F \subseteq(D-1) \circ E$, the statement follows from Theorem 4.9.

The proof can be extended to frameworks consisting of bodies, rods, bars, and hinges without difficulty.

Katoh and Tanigawa [16] showed that if each hinge is allowed to connect only two bodies, then each body can be realized as a rigid panel (i.e., a hyperplane). That is, a panel-hinge framework, which consists of rigid panels connected by hinges, is generically characterized by the counting condition of Corollary 5.1. A natural question is whether we can drop the restriction.

Problem 1. Let $G=(B, H ; E)$ be a bipartite graph satisfying the counting condition of Corollary 5.1. Is there a hinge-configuration $\boldsymbol{h}$ such that $(G, \boldsymbol{h})$ is an infinitesimally rigid panel-hinge framework?

Indeed, this problem was already discussed in, e.g., [34, 31, 40] and is unsolved even for the two-dimensional case. In [40], Whiteley presented a partial solution for the two-dimensional case.

In the context of combinatorial rigidity, three types of characterizations are typically considered: Maxwell-Laman-type counting conditions, Henneberg-type graph constructions, and tree-decompositions. In particular, tree-decompositions often provide very short proofs for combinatorial characterizations. See, e.g., [39, 33, 14]. It is hence natural to ask a tree-decomposition for identified body-hinge frameworks, which leads to Corollary 5.1.

Problem 2. Let $G=(B, H ; E)$ be a bipartite graph. Suppose there is an edge set $I \subseteq(D-1) \circ E$ such that $|I|=D|B|+(D-1)|H|-D$ and $|F| \leq D|B(F)|+$ $(D-1)|H(F)|-D$ for each nonempty $F \subseteq I$. Then, does $(D-1) \circ G$ contain $D$ edge-disjoint trees such that each vertex of $B$ is spanned by all of them and each vertex of $H$ is spanned by exactly $D-1$ trees among them?

The problem may be false since the problem of deciding whether a hypergraph contains $k$ edge-disjoint spanning connected subgraphs is NP-hard even for $k=2$ [5].

As for computational issue, $O\left(|V|^{2}\right)$ time algorithms are known for computing the rank of the counting (poly)matroids appearing in this paper (see, e.g., $[12,8,19,2]$ for more detail). Developing a subquadratic algorithm is indeed a challenging problem. 
6. Direction-rigidity. As a direct application of Dilworth truncation, we shall briefly discuss direction-rigidity of bar-joint frameworks.

Recall that a $d$-dimensional bar-joint framework is a pair $(G, \boldsymbol{p})$, where $G=(V, E)$ is a graph and $\boldsymbol{p}: V \rightarrow \mathbb{R}^{d}$. Each vertex represents a joint and each edge represents a bar which usually constraints the distance between two endpoints. As a variant of length-constraint, direction-constraint (and the mixture of length and direction constraints) has been considered in the literature (see, e.g., [41, 15, 29]). In [41], Whiteley showed a combinatorial characterization of direction-rigidity as a corollary of a combinatorial characterization of reconstructivity of pictures appeared in scene analysis (see, e.g., [40, 41, 42]). In this section we provide a direct proof of this characterization.

For a $d$-dimensional bar-joint framework $(G, \boldsymbol{p})$, an infinitesimal motion $\boldsymbol{m}: V \rightarrow$ $\mathbb{R}^{d}$ of $(G, \boldsymbol{p})$ under direction-constraint is an assignment of $\boldsymbol{m}(v) \in \mathbb{R}^{d}$ to each $v \in V$ such that $\boldsymbol{m}(u)-\boldsymbol{m}(v)$ is parallel to $\boldsymbol{p}(u)-\boldsymbol{p}(v)$ for any $u v \in E$, i.e., $\boldsymbol{m}(u)-\boldsymbol{m}(v)=$ $t(\boldsymbol{p}(u)-\boldsymbol{p}(v))$ for some $t \in \mathbb{R}$. Of course, the direction-constraint for each $u v \in E$ can be written as

$$
(\boldsymbol{m}(u)-\boldsymbol{m}(v)) \cdot \boldsymbol{\alpha}=0 \quad \text { for any } \boldsymbol{\alpha} \in \mathbb{R}^{d} \text { with }(\boldsymbol{p}(u)-\boldsymbol{p}(v)) \cdot \boldsymbol{\alpha}=0 .
$$

It is easy to observe that the space of infinitesimal motions of $(G, \boldsymbol{p})$ has dimension at least $d+1$; a linear combination of parallel transformations to $d$ directions and the dilation centered at the origin (see, e.g., [41, section 8] for more detail). We say that $(G, \boldsymbol{p})$ is direction-rigid if the dimension of the motion space is exactly $d+1$.

In this section, we shall use $V_{u}$ to denote a $d$-dimensional vector space associated with $u$ (which was $D$-dimensional in the preceding sections) and let $V_{V}$ denote the direct product of $V_{u}$ for all $u \in V$. Hence, $V_{V}$ is $d|V|$-dimensional in this case. For each $u v \in E$, let us define a $(d-2)$-dimensional flat of $\mathbb{P}\left(V_{V}\right)$ by

$$
A_{u v}(\boldsymbol{p})=\left\{\left[0, \cdots, 0, \stackrel{u}{\boldsymbol{\alpha}}, 0, \cdots, 0,{ }^{v} \boldsymbol{\alpha}, 0, \cdots, 0\right]: \boldsymbol{\alpha} \in \mathbb{R}^{d},(\boldsymbol{p}(u)-\boldsymbol{p}(v)) \cdot \boldsymbol{\alpha}=0\right\},
$$

and let $\mathcal{A}(\boldsymbol{p})=\left\{A_{e}(\boldsymbol{p}): e \in E\right\}$. Then, it is easy to see that direction-rigidity is characterized by the polymatroid $\mathcal{P} \mathcal{M}(\mathcal{A}(\boldsymbol{p}))$ in the sense that $(G, \boldsymbol{p})$ is directionrigid if and only if the rank of $\mathcal{P} \mathcal{M}(\mathcal{A}(\boldsymbol{p}))$ is equal to $d|V|-(d+1)$. The following theorem provides a combinatorial characterization of this polymatroid.

TheOREM 6.1. Let $f^{\prime}: 2^{E} \rightarrow \mathbb{Z}$ be an integer-valued monotone submodular function defined by

$$
f^{\prime}(F)=d|V(F)|-(d+1) \quad(F \subseteq E) .
$$

Then, for almost all joint-configurations $\boldsymbol{p}: V \rightarrow \mathbb{R}^{d}, \mathcal{P} \mathcal{M}(\mathcal{A}(\boldsymbol{p}))$ is equal to the polymatroid $\mathcal{P} \mathcal{M}_{f^{\prime}}(G)=\left(E, \hat{f}^{\prime}\right)$ induced by $f^{\prime}$.

Proof. We prove $\operatorname{rank}\left(\overline{\mathcal{A}_{F}(\boldsymbol{p})}\right)=\hat{f}^{\prime}(F)$ for any nonempty $F \subseteq E$ (see (2.1) for the definition of $\hat{f}$ ). The idea is exactly the same as the alternative proof of Laman's theorem by Lovász and Yemini [21].

Recall that $g=|V(\cdot)|-1$ is the monotone submodular function inducing graphic matroid. As mentioned in section 3.1, the union of $d$ copies of the graphic matroid is the matroid induced by $d g$ as well as the generic matroid associated with the family $\mathcal{A}=\left\{A_{e}: e \in E\right\}$ of flats

$$
A_{u v}=\left\{\left[0, \cdots, 0, \stackrel{u}{\boldsymbol{\alpha}}, 0, \cdots, 0,{ }^{v} \boldsymbol{\alpha}, 0, \cdots, 0\right]: \boldsymbol{\alpha} \in \mathbb{R}^{d}\right\} .
$$


In other words, $\mathcal{P} \mathcal{M}(\mathcal{A})=(E, \widehat{d g})$.

Denote $V=\left\{v_{1}, v_{2}, \ldots, v_{n}\right\}$. For $\boldsymbol{p}: V \rightarrow \mathbb{R}^{d}$, we define a hyperplane $H$ of $\mathbb{P}\left(V_{V}\right)$ by

$$
H=\left\{\left[x_{v_{1}}, x_{v_{2}}, \ldots, x_{v_{n}}\right]: \boldsymbol{x}_{v} \in V_{v}=\mathbb{R}^{d}, \sum_{v \in V} \boldsymbol{p}(v) \cdot \boldsymbol{x}_{v}=0\right\} .
$$

Then, observe $A_{e}(\boldsymbol{p})=A_{e} \cap H$ for any $e \in E$. Therefore, if we take $\boldsymbol{p}$ so that the set of coordinates of $\boldsymbol{p}$ is algebraically independent over $\mathbb{Q}$, we find that $\mathcal{P} \mathcal{M}(\mathcal{A}(\boldsymbol{p}))$ is obtained from $\mathcal{P} \mathcal{M}(\mathcal{A})$ by Dilworth truncation. By Theorem 2.2, we obtain, for any $F \subseteq E$,

$$
\begin{aligned}
& \operatorname{rank}\left(\overline{\mathcal{A}_{F}(\boldsymbol{p})}\right) \\
& \quad=\min \left\{\sum_{i}\left(\operatorname{rank}\left(\overline{\mathcal{A}_{F_{i}}}\right)-1\right): \text { a partition }\left\{F_{1}, \ldots, F_{k}\right\} \text { of } F\right\} \\
& \quad=\min \left\{\sum_{i}\left(\widehat{d g}\left(F_{i}\right)-1\right): \text { a partition }\left\{F_{1}, \ldots, F_{k}\right\} \text { of } F\right\} \\
& \quad=\min \left\{\sum_{i}\left(\left(\min \left\{\sum_{j} d g\left(F_{i, j}\right): \text { a partition of } F_{i}\right\}\right)-1\right): \text { a partition of } F\right\} \\
& \quad=\min \left\{\sum_{i}\left(d g\left(F_{i}\right)-1\right): \text { a partition }\left\{F_{1}, \ldots, F_{k}\right\} \text { of } F\right\} \\
& \quad=\min \left\{\sum_{i} f^{\prime}\left(F_{i}\right): \text { a partition }\left\{F_{1}, \ldots, F_{k}\right\} \text { of } F\right\}=\hat{f}^{\prime}(F),
\end{aligned}
$$

where we used $f^{\prime}(F)=d g(F)-1$. This completes the proof.

Let $(d-1) \circ G$ be the graph obtained from $G$ by replacing each edge by $(d-1)$ copies, and let $(d-1) \circ E$ be the edge set. Notice $f^{\prime}(e)=d-1$ for any $e \in E$. Hence, applying the same argument given in Lemma 4.1, it is not difficult to see that the rank of $\mathcal{P} \mathcal{M}_{f^{\prime}}(G)=\left(E, \hat{f}^{\prime}\right)$ is equal to the rank of $\mathcal{M}_{f^{\prime}}((d-1) \circ G)$, that is, the matroid on $(d-1) \circ E$ induced by $f^{\prime}$. Thus, Theorem 6.1 implies a combinatorial characterization of direction-rigidity of bar-joint frameworks proved by Whiteley [41].

Corollary 6.2 (Whiteley [41]). For almost all joint-configurations $\boldsymbol{p}: V \rightarrow \mathbb{R}^{d}$, $(G, \boldsymbol{p})$ is direction-rigid if and only if $(d-1) \circ G$ contains an edge subset $I \subseteq(d-1) \circ E$ satisfying the following counting conditions:

- $|I|=d|V|-(d+1)$;

- $|F| \leq d|V(F)|-(d+1)$ for any nonempty $F \subseteq E$.

Servatius and Whiteley [29] further proved a combinatorial characterization of generic rigidity of two-dimensional bar-joint frameworks having both length and direction constraints. It can be observed that the representation of the associated rigidity matrix can be obtained from the representation of the union of two copies of the graphic matroid by restricting some of rows to a generic hyperplane $H$ and the others to a hyperplane (determined by $H$ ). It is still unclear why Theorem 2.2 can be extended in this situation.

Appendix A. Description of bar-constraints. Here we give a note on how to obtain bar-constraints (3.3). This note also appears in [17, Appendix].

We can coordinatize the exterior product $\mathbb{R}^{d} \wedge \mathbb{R}^{d}$ as follows. For $a=\left(a_{1}, a_{2}, \ldots\right.$, $\left.a_{d}\right) \in \mathbb{R}^{d}$ and $b=\left(b_{1}, b_{2}, \ldots, b_{d}\right) \in \mathbb{R}^{d}$,

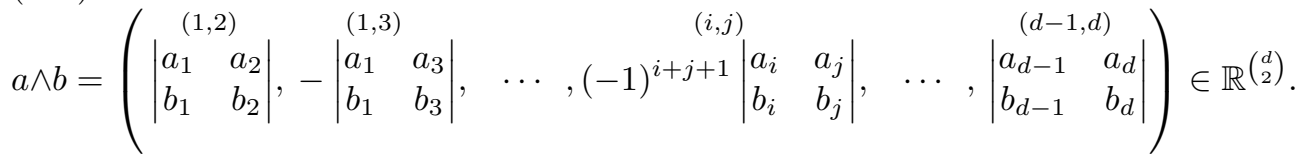

Suppose we are given rigid bodies $B_{1}$ and $B_{2}$ in $\mathbb{R}^{d}$, which can be identified with a pair $\left(p_{i}, M_{i}\right)$ of a point $p_{i} \in \mathbb{R}^{d}$ and an orthogonal matrix $M_{i} \in S O(d)$ for 
each $i=1,2$. Namely, each $\left(p_{i}, M_{i}\right)$ is a local Cartesian coordinate system for each body. We consider a situation, where the bodies $B_{1}$ and $B_{2}$ are connected by a bar. We denote the endpoints of the bars by $p_{1}+M_{1} q_{1}$ and $p_{2}+M_{2} q_{2}$, where $q_{i}$ is the coordinate of each endpoint (joint) in the coordinate system of each body.

The constraint by the bar can be written by

$$
\left\langle p_{2}+M_{2} q_{2}-p_{1}-M_{1} q_{1}, p_{2}+M_{2} q_{2}-p_{1}-M_{1} q_{1}\right\rangle=\ell^{2}
$$

for some $\ell \in \mathbb{R}$. If we take the differentiation with variables $p_{i}$ and $M_{i}$, we get

$$
\left\langle p_{2}+M_{2} q_{2}-p_{1}-M_{1} q_{1}, \dot{p}_{2}+\dot{M}_{2} q_{2}-\dot{p}_{1}-\dot{M}_{1} q_{1}\right\rangle=0 .
$$

We may simply assume $p_{i}=0$ and $M_{i}=I_{d}$. Then by setting $h=q_{2}-q_{1}$ and $\dot{M}_{i}=A_{i}$ with a skew-symmetric matrix $A_{i}$,

$$
\left\langle h, \dot{p}_{2}+A_{2} q_{2}-\dot{p}_{1}-A_{1} q_{1}\right\rangle=0 .
$$

Also we denote a skew-symmetric matrix $A$ by

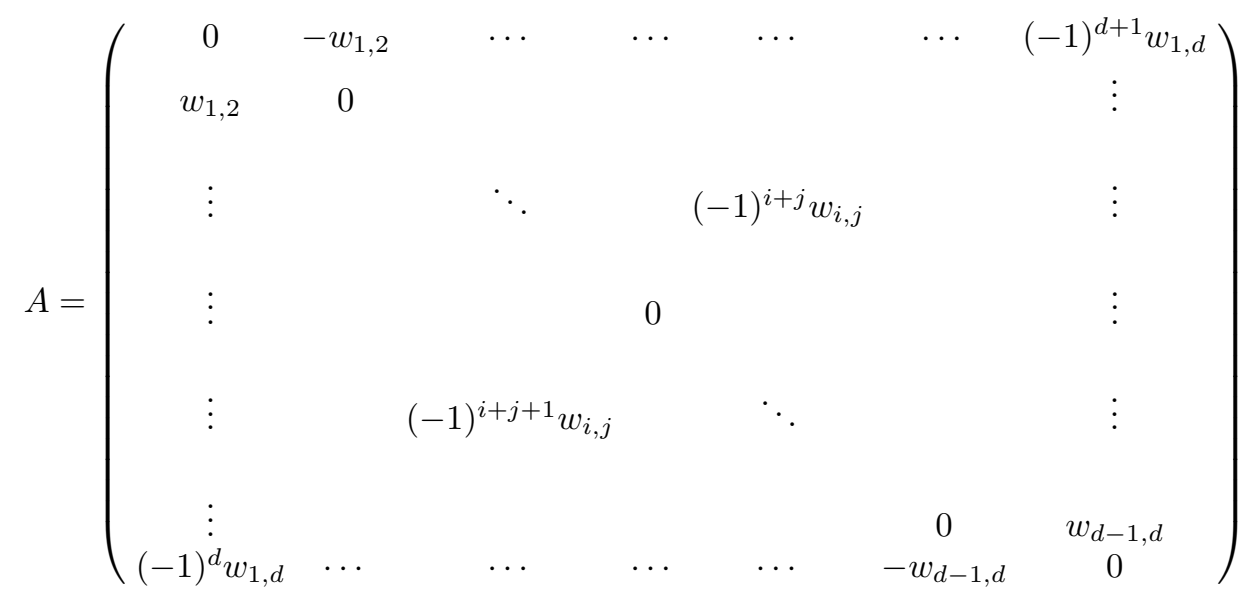

and let $w=\left(\begin{array}{llll}w_{1,2} & w_{1,3} & \cdots & w_{d-1, d}\end{array}\right) \in \mathbb{R}^{\left(\begin{array}{l}d \\ 2\end{array}\right)}$. Then, for any $h \in \mathbb{R}^{d}$ and $q \in \mathbb{R}^{d}$, we have

$$
\langle h, A q\rangle=\langle q \wedge h, w\rangle .
$$

Therefore, we can simply describe the infinitesimal bar-constraint (A.4) by

$$
\left\langle q_{2}-q_{1}, \dot{p}_{2}-\dot{p}_{1}\right\rangle+\left\langle q_{2} \wedge q_{1}, w_{2}-w_{1}\right\rangle=0,
$$

where $w_{1} \in \mathbb{R}^{\left(\begin{array}{l}d \\ 2\end{array}\right)}$ and $w_{2} \in \mathbb{R}^{\left(\begin{array}{l}d \\ 2\end{array}\right)}$ denote the $\left(\begin{array}{l}d \\ 2\end{array}\right)$-dimensional vectors corresponding to $A_{1}$ and $A_{2}$, respectively.

We call a pair $\left.s_{i}=\left(w_{i}, p_{i}\right) \in \mathbb{R}_{\left(\begin{array}{l}d \\ 2\end{array}\right)}\right) \times \mathbb{R}^{d}$ a screw motion, which can be identified with a vector in $\bigwedge^{d-1} \mathbb{R}^{d+1}$. Using the homogeneous coordinate of $q_{i}$ in $\mathbb{P}^{d}$, (A.6) is written as

$$
\left.\left\langle\left(q_{2}, 1\right) \wedge\left(q_{1}, 1\right)\right), s_{2}-s_{1}\right\rangle=0,
$$

where $\left[\left(q_{2}, 1\right) \wedge\left(q_{1}, 1\right)\right]$ is the Plücker coordinate of the corresponding bar. 


\section{REFERENCES}

[1] M. Barnabei, A. Brini, And G. Rota, On the exterior calculus of invariant theory, J. Algebra, 96 (1985), pp. 120-160.

[2] A. Berg And T. Jordán, Algorithms for graph rigidity and scene analysis, in Proceedings of the 11th Annual European Symopsium on Algorithms (ESA), Lecture Notes in Comput. Sci. Springer, New York, 2003, 2832 pp. 78-89.

[3] J. Edmonds and G. C. Rota, Submodular set functions, in proceedings of Waterloo Conference on Combinatorics, 1966.

[4] A. Frank, Connections in Combinatorial Optimization, Oxford Lecture Ser. Math. Appl., Oxford University Press, Oxford, UK, 2011.

[5] A. Frank, T. KirÁly, and M. Kriesell, On decomposing a hypergraph into $k$ connected sub-hypergraphs, Discrete Appl. Math., 131 (2003), pp. 373-383.

[6] A. Frank and L. Szegö, Constructive characterizations for packing and covering with trees, Discrete Appl. Math., 131 (2003), pp. 347-371.

[7] S. Fujishige, Submodular Functions and Optimization, Annals of Discrete Mathematics, 2nd ed., Elsevier, New York, 2005.

[8] H. Gabow and H. Westermann, Forests, frames, and games: Algorithms for matroid sums and applications, Algorithmica, 7 (1992), pp. 465-497.

[9] H. GlucK, Almost all simply connected closed surfaces are rigid, in Geometric Topology, Lecture Notes in Math., Springer 438, New York, 1975, pp. 225-240.

[10] B. Hassett, Introduction to Algebraic Geometry, Cambridge University Press, London, 2007.

[11] W. V. D. Hodge And D. Pedoe, Methods of Algebraic Geometry, vol. 1, Cambridge University Press, London, 1994.

[12] H. Imai, Network flow algorithms for lower truncated transversal polymatroids, J. Oper. Res. Soc. Japan, 26 (1983), pp. 186-210.

[13] B. JACKSON AND T. JORDÁN, On the rank function of the 3-dimensional rigidity matroid, Internat. J. Comput. Geom. Appl., 16 (2006), pp. 415-429.

[14] B. JACKSON AND T. JoRDÁn, The generic rank of body-bar-and-hinge frameworks, European J. Combin., 31 (2009), pp. 574-588.

[15] B. JaCKSON AND T. JoRDÁn, Globally rigid circuits of the direction-length rigidity matroid, J. Combin. Theory Ser. B, 100 (2010), pp. 1-22.

[16] N. Katoh and S. Tanigawa, A proof of the molecular conjecture, Discrete Comput. Geom., 45 (2011), pp. 647-700.

[17] N. Katoh AND S. TANigawa, Rooted-tree decompositions with matroid constraints and infinitesimal rigidity of frameworks with boundaries, arXiv:1109.0787v1, 2011.

[18] G. Laman, On graphs and rigidity of plane skeletal structures, J. Engrg. Math., 4 (1970), pp. 331-340.

[19] A. Lee And I. Streinu, Pebble game algorithms and sparse graphs, Discrete Math., 308 (2008), pp. $1425-1437$.

[20] L. LovÁsz, Flats in matroids and geometric graphs, in Combinatorial Surveys: Proceedings of the Sixth British Combinatorial Conference, Academic Press, 1977, pp. 45-86.

[21] L. Lovász And Y. Yemini, On generic rigidity in the plane, SIAM J. Algebraic Discrete Methods, 3 (1982), pp. 91-98.

[22] J. H. MASON, Matroids as the study of geometrical configurations, in Higher Combinatorics (Proceedings of the NATO Advanced Study Institute, 1976), M. Aligner ed., D. Reidel, Boston, 1977, pp. 133-176.

[23] J. H. MASOn, Glueing matroids together: A study of dilworth truncations and matroid analogues of exterior and symmetric powers, in Algebraic Methods in Graph Theory, vol. II (Colloquium Szeged, 1978), L. Lovász and V. T. Sós, eds., North-Holland, Amsterdam, 1981 , pp. 519-561.

[24] C. Nash-Williams, Decomposition of finite graphs into forests, J. London Math. Soc., 39 (1964), p. 12.

[25] E. Nevo, On embeddability and stresses of graphs, Combinatorica, 27 (2007), pp. 465-472.

[26] J. OxLeY, Matroid theory, Oxford University Press, New York, 1992.

[27] J. Pym and H. Perfect, Submodular functions and independence structures, J. Math. Anal. Appl., 30, pp.1-31, (1970).

[28] A. Schrijver, Combinatorial Optimization: Polyhedra and Efficiency, Springer, New York, 2003.

[29] B. Servatius and W. Whiteley, Constraining plane configurations in computer-aided design: Combinatorics of directions and lengths, SIAM J. Discrete Math., 12 (1999), pp. 136-153.

[30] T. TAY, Rigidity of multi-graphs. I: Linking rigid bodies in n-space, J. Combin. Theory Ser. B, 36 (1984), pp. 95-112. 
[31] T. TAY, Linking $(n-2)$-dimensional panels in $n$-space II: $(n-2,2)$-frameworks and body and hinge structures, Graphs Combin., 5 (1989), pp. 245-273.

[32] T. TAY, Linking $(n-2)$-dimensional panels in $n$-space I: $(k-1, k)$-graphs and $(k-1, k)$-frames, Graphs Combin., 7 (1991), pp. 289-304.

[33] T. TAY, A new proof of Laman's theorem, Graphs Combin., 9 (1993), pp. 365-370.

[34] T. S. TAY AND W. Whiteley, Recent advances in the generic rigidity of structures, Structural Topology, 9 (1984), pp. 31-38.

[35] T. S. TAy And W. Whiteley, Generating isostatic graphs, Structural Topology, 11 (1985), pp. 21-68.

[36] N. White, Grassmann-Cayley algebra and robotics, J. Intelligent and Robotic Systems, 11 (1994), pp. 91-107.

[37] N. White AND W. Whiteley, The algebraic geometry of motions of bar-and-body frameworks, SIAM J. Algebraic Discrete Methods, 8 (1987), pp. 1-32.

[38] W. Whiteley, Infinitesimal motions of a bipartite framework, Pacific J. Math, 110 (1984), pp. 233-255.

[39] W. WhITELEY, The union of matroids and the rigidity of frameworks, SIAM J. Discrete Math., 1 (1988), pp. 237-255.

[40] W. Whiteley, A matroid on hypergraphs with applications in scene analysis and geometry, SIAM J. Discrete Math., 4 (1989), pp. 75-95.

[41] W. Whiteley, Some matroids from discrete applied geometry, Contemp. Math., 197 (1996), pp. 171-312.

[42] W. Whiteley, Rigidity and scene analysis, in Handbook of Discrete and Computational Geometry, J. E. Goodman and J. O'Rourke, eds., CRC Press, Boca Raton, FL, 2004, pp. $1327-1354$

Copyright (C) by SIAM. Unauthorized reproduction of this article is prohibited. 